\title{
Effects of aging on value-directed modulation of semantic network activity during verbal learning
}

\author{
Michael S. Cohen $^{\mathrm{a}, \mathrm{d}, *}$, Jesse Rissman ${ }^{\mathrm{a}, \mathrm{b}}$, Nanthia A. Suthana ${ }^{\mathrm{a}, \mathrm{b}, \mathrm{c}}$, Alan D. Castel ${ }^{\mathrm{a}}$, Barbara J. Knowlton ${ }^{\mathrm{a}}$ \\ a Department of Psychology, University of California, Los Angeles, USA \\ b Department of Psychiatry \& Biobehavioral Sciences, University of California, Los Angeles, USA \\ c Department of Neurosurgery, University of California, Los Angeles, USA \\ d Department of Psychology, Northwestern University, Evanston, IL, USA
}

\section{A R T I C L E I N F O}

\section{Article history:}

Received 13 March 2015

Accepted 28 July 2015

Available online 2 August 2015

\section{Keywords:}

Aging

Metacognitive control

Learning

Prefrontal cortex

fMRI

\begin{abstract}
A B S T R A C T
While impairments in memory recall are apparent in aging, older adults show a remarkably preserved ability to selectively remember information deemed valuable. Here, we use fMRI to compare brain activation in healthy older and younger adults during encoding of high and low value words to determine whether there are differences in how older adults achieve value-directed memory selectivity. We find that memory selectivity in older adults is associated with value-related changes in activation during word presentation in left hemisphere regions that are involved in semantic processing, similar to young adults. However, highly selective young adults show a relatively greater increase in semantic network activity during encoding of high-value items, whereas highly selective older adults show relatively diminished activity during encoding of low-value items. Additionally, only younger adults showed value-related increases in activity in semantic and reward processing regions during presentation of the value cue preceding each to-be-remembered word. Young adults therefore respond to cue value more proactively than do older adults, yet the magnitude of value-related differences in cue period brain activity did not predict individual differences in memory selectivity. Thus, our data also show that age-related reductions in prestimulus activity do not always lead to inefficient performance.
\end{abstract}

(c) 2015 Elsevier Inc. All rights reserved.

\section{Introduction}

People often need to remember information that has been labeled as important, at the expense of information deemed to be less important. Some information may be especially important to learn, because doing so will yield a subsequent reward. A number of neuroimaging studies (e.g., Adcock et al., 2006; Düzel et al., 2010; Bunzeck et al., 2012; Wolosin et al., 2012; Gruber et al., 2014) have examined how dopaminergic reward-sensitive brain regions interact with the hippocampus to enhance intentional encoding of motivationally significant items (for review, see Shohamy and Adcock, 2010). In our recent study (Cohen et al., 2014), we examined how reward affects verbal memory under conditions that encourage the adoption of different encoding strategies for high-value and low-value items. We found that the degree to which value affects memory on a subsequent free recall test correlates with value-related differences in activity in a largely left-lateralized network of brain regions: L ventrolateral prefrontal cortex (VLPFC)/inferior frontal gyrus (IFG), L posterior lateral temporal cortex, bilateral posterior medial prefrontal cortex/pre-supplementary motor area (pre-SMA), and L caudate. All of these regions have been

\footnotetext{
* Corresponding author at: Department of Psychology, Northwestern University, 2029 Sheridan Rd., Evanston, IL 60208, USA.

E-mail address: michael.cohen@northwestern.edu (M.S. Cohen).
}

associated with semantic processes (Binder et al., 2009), which we believe are being modulated as part of a conscious strategy to deeply encode the high value words (Galli, 2014). Thus, our results-collected from a sample of healthy young adults-were the first to emphasize that reward can modulate memory via the intentional, differential use of effective encoding strategies, beyond its role in activating the mesolimbic dopamine system.

Effects of aging on the relationship between item value and memory performance

Another important question is how the cognitive and neural mechanisms by which value affects memory encoding change across the lifespan. Prior work has not directly addressed how the neural mechanisms of this process change in older adults. However, there is some relevant behavioral work. For instance, Spaniol et al. (2014) presented older and younger adults with a version of the task paradigm used by Adcock et al. (2006), in which value is believed to affect memory predominantly by increasing activation in the mesolimbic dopamine system. Spaniol and colleagues found no interaction between age and value on hit rates in a subsequent recognition test, meaning that highreward conditions led to similar improvements in memory across age groups, despite overall poorer memory in older adults, and despite 
evidence for age-related neural degradation in dopamine-producing regions of the midbrain (e.g., Bunzeck et al., 2007).

Castel et al. (2002, 2009) have examined how age affects valuebased modulation of memory in a context in which subjects must prioritize recall of high value information (see also Hayes et al., 2013). Although older adults do recall fewer items than young adults in these studies, their selectivity index, a measure of how strongly value affects memory, tends to be as high, or in some cases even higher, than that seen in young adults. Thus, regardless of the mechanism by which value affects memory, healthy older adults appear to retain the ability to remember the things that are most important to the task at hand even as their overall memory gets worse.

\section{Temporal shifts in brain activity with aging}

One key question that we address in the present study pertains to the timing of value-related activity changes. Namely, are activity modulations triggered immediately in response to the cue stimuli that indicate the value of an upcoming word, or do these modulations occur later, during presentation of the word itself? We are also interested in understanding how these temporal patterns may change with age. Adcock et al. (2006) found increased activity in reward-sensitive regions and in medial temporal lobe (MTL) structures during high-value cues, relative to low-value cues, as well as a subsequent memory effect for high-value items in particular. Although there were value-related differences in MTL activity during stimulus presentation, they did not find value effects in reward regions during presentation of the to-beremembered picture stimuli. The importance of activity in dopaminergic regions, particularly during the cue period, follows from animal work showing that exposure to dopamine agonists a few minutes prior to stimulus presentation can lower the threshold for long-term potentiation (LTP) in the hippocampus (e.g., Li et al., 2003). Thus, there is a mechanistic explanation for why strengthening of encoding would specifically occur for items presented after a reward cue (although see Murayama and Kitagami, 2014, for an example of putative dopamine-driven memory enhancement in humans when the memory stimulus is presented prior to the reward).

Other prior research has shown that brain activity in MTL and neocortical regions in response to a cue indicating how to encode an upcoming item can also differ as a function of subsequent memory status. One such study used electroencephalography (EEG) to measure event-related potentials (ERP) evoked in response to cue stimuli signaling the need for an imminent semantic decision about an upcoming word (Otten et al., 2006). The magnitude of these pre-stimulus ERP effects in frontal and posterior regions of the cortex was linked to the subsequent mnemonic fate of these items. Gruber and Otten (2010) examined how pre-stimulus ERP effects are affected by reward, and found a diffusely-localized pattern of more positive ERP activity during high-value cues relative to low-value cues, particularly when the high-value words that followed a given cue were later recognized with high confidence. Pre-stimulus activity was not associated with better memory when the cue was lowvalue, however, suggesting that these pre-stimulus effects are sensitive to motivation. Other studies have used functional magnetic resonance imaging (fMRI) to examine effects of pre-stimulus activity on subsequent memory, allowing for better localization of where the relevant activity is taking place. Subsequent memory effects in response to pre-stimulus cues have been shown bilaterally in MTL (Park and Rugg, 2010), as well as in lateral prefrontal cortex (PFC) and lateral/medial parietal cortex (Addante et al., 2015). These authors have suggested that subsequent memory effects based on pre-stimulus activity likely result from the adoption of a "preparatory set", in which the brain is more prepared to engage effective encoding operations once the to-be-remembered item appears.

There is reason to believe that older adults might be less able to benefit from pre-stimulus cues than young adults. For instance, Bollinger et al. (2010) showed that young adults tend to show more fusiform face area (FFA) activity, and more connectivity between FFA and fronto-parietal control regions, in response to a cue that the to-beencoded item about to appear is a face, compared to when no informative cue is presented or when the cue indicates that a scene is about to appear. Memory for face stimuli, in response to an immediate working memory probe and also after a 30-minute delay, was better when such a cue was presented, and the degree of cue-related memory benefit was correlated with the degree of enhanced connectivity between FFA and specific fronto-parietal regions. Thus, the increase in FFA/ fronto-parietal connectivity, induced by presentation of the informative cue, appears to have strengthened encoding in young adults. Older adults did not show a change in FFA activity or connectivity in response to the cue, nor did they show memory benefits in response to the cue (Bollinger et al., 2011). Based on these findings, Bollinger et al. (2011) proposed an "expectation deficit hypothesis of cognitive aging," suggesting that an inability to utilize informative cues underlies some aging-related cognitive deficits.

Other work has suggested that older adults tend to rely more heavily on activity later in a trial to compensate for a lack of activity in response to an earlier cue. Dew et al. (2012) found such a pattern in MTL and left dorsolateral PFC (DLPFC) regions during a memory retrieval task. Specifically, young adults show more activity than older adults during a pre-stimulus cue indicating what type of stimulus will need to be retrieved, while older adults show more activity later in the trial, during memory retrieval. Dew et al. refer to this pattern as an Early to Late Shift in Aging, or ELSA.

The results shown by Dew et al. could be considered an extension of the Dual Modes of Control theory (Braver, 2012; Braver et al., 2007, 2009), which has been influential in the domain of cognitive control, to the domain of memory retrieval. Braver et al. (2009) found that young adults are more likely to keep a task set in working memory in anticipation of relevant stimuli appearing later, referred to as a proactive mode of cognitive control. By contrast, older adults tend to use a more reactive mode of cognitive control, waiting to engage cognitive control mechanisms until the appearance of a stimulus for which control is required. Based on this prior literature, we might expect that when participants encode to-be-remembered words, as in the present study, young adults will be more likely to show value-related changes in brain activity during the value cues that precede the words, while older adults will only show value-related differences in activity after the word appears.

\section{Spatial shifts in brain activity with aging}

Another important focus of the present study is on how aging might lead to shifts in the localization of value-related differences in encodingrelated activity, either in an attempt to compensate for aging-related deficits, or as a consequence of those deficits. Logan et al. (2002) found, for instance, that older adults typically show less activity in the left VLPFC than young adults when asked to remember words. However, the difference largely disappears when the depth of semantic processing is controlled by the experimenter. Logan et al. (2002) also found that unlike young adults, for whom VLPFC activity was largely leftlateralized during word encoding, older adults showed nearly as much activity in right VLPFC as in left VLPFC when they did engage this area. Logan et al. concluded that the right hemisphere activation was due to less efficient processing. This interpretation, known as dedifferentiation, implies that an aging-related reduction in lateralization and/or neuroanatomical specialization contributes to deficits in cognitive functioning (see also Li et al., 2001).

Cabeza (2002), however, proposed that activity in the contralateral hemisphere is an attempt to compensate for degradation in the areas in which processing is typically performed. There is striking evidence in favor of compensation in certain contexts. For instance, Cabeza et al. (2002) used a retrieval task in which the key contrast showed activity 
in right anterior PFC and right DLPFC in young adults. In lowerfunctioning older adults, activity was still entirely right-lateralized. High-functioning older adults showed a different pattern, however, recruiting left anterior PFC in addition to right anterior PFC.

During encoding, as opposed to at retrieval, activity tends to be more left-lateralized in young adults, and the evidence has been more mixed as to whether reduced prefrontal laterality in older adults (such as that observed by Logan et al., 2002) reflects compensation. Rosen et al. (2002) found support for the compensation account; they compared semantic encoding blocks to shallow encoding blocks, and found greater enhancement of right VLPFC activity on semantic blocks in higher-performing older adults, relative to young adults and lowerperforming older adults. At the same time, others (e.g., Rossi et al., 2004) have found evidence that activity in the contralateral hemisphere is not beneficial for left-hemisphere-dominant encoding tasks, but it does support performance in right-hemisphere-dominant retrieval tasks.

A somewhat different perspective on how aging affects PFC function has been proposed by Rajah and D'Esposito (2005); specifically, they suggest that effects of aging vary by region. In VLPFC, increased bilateral recruitment does not seem to enhance performance (e.g., Logan et al., 2002), and thus those activations likely reflect either dedifferentiation or failed attempts at compensation. However, there does not appear to be a primary functional deficit in this region in older adults; when VLPFC is properly engaged, older adults can perform successfully on tasks relying upon this region. By contrast, in dorsal and anterior PFC, there is a distinction across hemispheres. In the right hemisphere, there seems to be a true functional deficit with aging; even when these regions are activated, they do not contribute to task performance in older adults. In the left hemisphere, by contrast, more dorsal and anterior PFC regions seem to be able to compensate for dysfunction in the homologous right hemisphere regions.

\section{The present study}

In the present fMRI study, we examine whether brain activation associated with value-related selectivity in healthy older adults differs from that in younger adults in terms of its temporal and spatial pattern. While older and younger adults often exhibit a similar degree of selectivity on the value-directed remembering task, it is unclear if the neural mechanisms supporting selectivity are the same. Because selective remembering of valuable information is relatively preserved in older adults, this paradigm is a particularly appropriate one in which to examine neural mechanisms of compensation in the aged brain.

\section{Materials and methods}

\section{Participants}

Twenty-five older adults were recruited to participate in the study via flyers posted at the UCLA Medical Center, and via flyers and newsletter postings in the broader West Los Angeles and San Fernando Valley communities. Data from two participants were excluded from all analyses due to neurological abnormalities detected during scanning (one cavernoma, one meningioma).

The remaining 23 older adult participants (mean age $=68.70$ years, $\mathrm{SD}=5.72$ years, range $=60-80$ years; 13 female) were all righthanded native English speakers with no neurological abnormalities. In addition, none of these individuals reported currently taking psychoactive medication for a major psychiatric disorder. All participants scored at least a 27 on a version of the Mini-Mental State Exam (Folstein et al., 1975), and had either normal or corrected-to-normal vision. Written informed consent was obtained from each participant, and all procedures were approved by UCLA's Medical Institutional Review Board. Participants were paid a base rate of $\$ 15 / \mathrm{h}$, plus additional bonus money based on performance on two additional tasks that were conducted during the same session; these two tasks were performed after the main experiment and are not reported here. For two participants, complete data sets were only available for 4 out 5 functional scanning runs (due to time constraints and technical difficulties, respectively). Finally, behavioral data from the practice session were unavailable for 2 individuals.

The young adult comparison group for this study $(\mathrm{N}=20)$ has been described previously (Cohen et al., 2014). They met similar inclusion criteria as the older adults, with the following demographics: mean age $=21.65$ years, $\mathrm{SD}=3.66$, age range $=18-30$ years, 11 females.

\section{Task stimuli and behavioral procedures}

The task paradigm was identical to that used in our previous study of young adults (Cohen et al., 2014). Each trial began with a cue stimulus indicating how many points could be earned if the ensuing word stimulus was later recalled in a memory test. Value cues were presented as digits inside of a gold "coin", and values could be either high $(10,11$, or 12 points) or low $(1,2$, or 3 points). The cue $(2 \mathrm{~s})$ was followed by a jittered fixation cross (25\% 3 s, 25\% 4.25 s, 25\% 5.5 s, 25\% 6.75 s). Then, the to-be-remembered word was presented $(3.5 \mathrm{~s})$ followed by another fixation $(1.5 \mathrm{~s})$. Finally, participants were presented with a vowel-consonant judgment task for 3.75-8.75 s. In this task, 2-6 letters (50\% of trials: 2 letters, $25 \% 4$ letters, $25 \% 6$ letters) were presented, one at a time, in a pseudo-random order, with an approximately equal number of vowels and consonants presented. Each letter was shown for a duration of $1 \mathrm{~s}$, followed by a $0.25 \mathrm{~s}$ fixation between letters, and a $1.5 \mathrm{~s}$ blank screen after the final letter. The active baseline period was intended to partially constrain rehearsal to the time period when the word was actually on the screen.

Each list included 24 different words. Half of these words were arbitrarily assigned a high point value and the other half were assigned a low point value (with 4 words at each specific value level); value assignment was counterbalanced across participants. All words were drawn from clusters 6 and 7 of the Toglia and Battig (1978) word norms. All were 4-8 letter nouns, rated as highly familiar (range 5.5-7 on a 1-7 scale), moderate to high on concreteness and imagery (range 4-6.5 on a 1-7 scale), and moderate in pleasantness (range $2.5-5.5$ on a 1-7 scale). Each list began with $10 \mathrm{~s}$ of fixation and ended with an extra $15 \mathrm{~s}$ of the vowel-consonant task. Within about 10-20 s after the end of each scanning run, the recall test began, and the participant was given $90 \mathrm{~s}$ to recall as many words as possible from the preceding list. Immediately after recall was complete, the experimenter scored the test, and gave feedback on the point score earned for that list. Participants completed five such study-test cycles in the scanner, with one list per scanning run; list order was counterbalanced across participants.

Prior to scanning, participants were given detailed instructions about the value-directed memory task, and then completed six practice items, followed by two full practice lists. Each of the two full practice lists included recall tests with feedback. Prior work has shown that selectivity is typically stronger on the third and subsequent lists than on the first two lists (Ariel and Castel, 2014; Castel, 2008; McGillivray and Castel, 2011). Thus, we assumed that by presenting two full lists prior to scanning, strategy use would be relatively well established and consistent in the scanner.

Following the scan, we gave participants a paper-and-pen questionnaire that included open-ended questions about what encoding strategy they used, whether the strategy that they used changed across lists, and whether they did anything differently for the high-value items. We also asked whether they rehearsed words during the fixation period between cue and target word and/or during the vowel/consonant task, and if so what type of words they rehearsed (e.g., high-value words, most recent words, etc.). Based on the responses to these questions, it was possible to gain a rough measure of whether and how strongly item value affected explicit encoding processes, as well as the type of 
encoding strategy a given participant generally used (e.g., deep, meaning-based encoding vs. shallower rote-rehearsal type strategies). An analysis of how these questionnaire responses correspond to individual differences in the behavioral data is included in the Supplemental Results.

We computed each participant's memory selectivity index for each list using the formula [(actual score - chance score) / (ideal score chance score)], as described in prior literature (Castel et al., 2002; Watkins and Bloom, 1999). We then averaged the selectivity indices across all scanned lists to yield a single score, weighting the average by the number of items recalled on each list. We used this weighting procedure, identical to that used by Watkins and Bloom (1999), because older adults occasionally recalled only a small number of items on some of the lists, and thus we felt that giving a reduced weight to these lists would yield a more accurate measure of selectivity. ${ }^{1}$

\section{Scanning procedure}

T2*-weighted echoplanar (EPI) images sensitive to blood oxygenation level dependent (BOLD) contrast were collected using a $3 \mathrm{~T}$ Siemens Tim Trio MRI scanner at the UCLA Staglin IMHRO Center for Cognitive Neuroscience. Each 179-volume functional run lasted approximately 7.5 min; five such runs were acquired for each participant. Each functional volume consisted of 45 interleaved axial slices, TR = $2500 \mathrm{~ms}, \mathrm{TE}=25 \mathrm{~ms}$, flip angle $=75^{\circ}$, slice thickness $=3.0 \mathrm{~mm}$ (no gap), in-plane resolution $=3.0 \times 3.0 \mathrm{~mm}$, matrix $=64 \times 64$, FoV $=$ $192 \mathrm{~mm}$. In addition, we collected matched-bandwidth T2-weighted coplanar structural scans to use as an intermediate step in spatial registration. We also collected a high-resolution structural scan (MPRAGE), using the following parameters: $\mathrm{TR}=1900 \mathrm{~ms}$, $\mathrm{TE}=3.26 \mathrm{~ms}$, flip angle $=9^{\circ}, 176$ slices, $1 \mathrm{~mm}^{3}$ voxels, $18.2 \%$ slice oversampling, FoV $=250 \mathrm{~mm}$, with GRAPPA acceleration. To minimize head movement during scanning, we placed extra cushions between the subject's head and the coil. Stimuli were presented using E-Prime 2.0 software (Psychology Software Tools, Pittsburgh, PA), and images were shown via either a custom-built MR-compatible rear projection system, or via MR-compatible goggles (Resonance Technology, Inc.).

\section{fMRI data analysis}

\section{Preprocessing}

Analyses of EPI data were carried out using FEAT v5.98 (fMRI Expert Analysis Tool), as implemented in FSL v4.1.9 (www.fmrib.ox.ac.uk/fsl). We corrected for head motion using MCFLIRT (FMRIB's motion correction linear image registration tool; Jenkinson et al., 2002), and also used the fsl_motion_outliers script to detect and censor any volumes with excessive head motion. Non-brain tissue was removed using BET (Brain Extraction Tool; Smith, 2002). Grand-mean intensity normalization was applied to the 4D dataset from each run based on a multiplicative scaling factor. We applied a Gaussian kernel of $5 \mathrm{~mm}$ FWHM for spatial smoothing, and for temporal filtering, a high-pass filter was applied to remove low-frequency noise using a Gaussian-weighted least-squares straight-line fitting with a sigma of $100 \mathrm{~s}$. Temporal autocorrelation was corrected for using prewhitening as implemented by FILM (FMRIB's improved linear model; Woolrich et al., 2001). Functional images were registered to a coplanar structural scan, and these in turn were registered to a high-resolution MPRAGE scan using FLIRT (FMRIB's Linear Image Registration Tool) linear registration. All images were then transformed to standard Montreal Neurological Institute (MNI) space

\footnotetext{
${ }^{1}$ Note that in our earlier paper (Cohen et al., 2014) we used an unweighted average of selectivity index. We switch to using a weighted average for both age groups in the present paper. In any case, the distinction is slight, as weighted and unweighted selectivity indices are highly correlated between individuals: $r=.996$ in young adults, and $r=.965$ in older adults.
}

according to nonlinear parameters estimated by warping the subject's MPRAGE image to a T1 atlas using FNIRT (FMRIB's Non-linear Image Registration Tool).

\section{Analysis}

We included four different event types in the statistical model for our primary general linear model (GLM) analysis: high-value cue period, high-value word-encoding period, low-value cue period, and low-value word-encoding period. The cue period was defined based on the time period in which each value cue was on-screen, $2 \mathrm{~s}$ in duration, convolved with a double-gamma hemodynamic response function (HRF). The word-encoding period was defined as a separate event, based on the time period in which the to-be-learned word was onscreen, $3.5 \mathrm{~s}$ in duration, convolved with a double-gamma HRF. Temporal derivatives were included in the model for all four event types. Motion regressors generated by MCFLIRT and regressors coding for any motion outlier TRs were also included in the model as covariates of no interest.

A first-level GLM analysis was carried out separately for each run, followed by a second-level fixed-effects analysis, combining parameter estimates across all runs and creating a set of linear contrasts. Our primary contrasts of interest compared the BOLD signal during highvalue vs. low-value items, looking separately at the cue period data and the word-encoding period data. For whole-brain analyses across subjects, we used the FLAME stages 1 and 2 mixed effects model in FSL, with automatic outlier detection. Clusters were determined using a voxel-level threshold of $Z>2.3$, with a cluster-corrected significance level of $p<.05$. Cortical surface renderings were created using Caret v5.65 (http://brainvis.wustl.edu; Van Essen et al., 2001) on the inflated Conte69 atlas in FNIRT space (Van Essen et al., 2012), with FSL activation maps transformed from volume to surface space using Caret's interpolated voxel algorithm. Activation peaks noted in the tables were a subset of the local maxima generated for each contrast by FSL's "cluster" command, with a minimum distance of $10 \mathrm{~mm}$ between peaks. Labels were determined using the Harvard-Oxford structural atlas and other relevant brain atlases (e.g., Talairach and Tournoux, 1988; Brodmann, 1909), and redundant peaks were eliminated. To search the whole brain for correlations between behavioral measures (e.g., selectivity index) and changes in BOLD signal, we included the behavioral measure as an EV in an FSL group-level model, in addition to the group mean. For region of interest (ROI) analyses, we computed Pearson correlation coefficients across participants using each individual's mean selectivity index and the mean contrast of parameter estimate (COPE) values for a given contrast in a given ROI for each participant.

To examine age differences in the effects of value throughout the brain, we included young adults and older adults in a single grouplevel analysis, assigning young and older adults to different regressors, and also labeling them as belonging to separate variance groups. Because we were specifically interested in how aging impacts the expression of value-induced activity modulations, we masked the resulting contrasts to only include voxels that showed corresponding effects in one or both age groups; voxels that failed to show significant effects in either age group were excluded. By always conducting across-group contrasts of within-subject COPE values, we avoided direct comparison of BOLD signal levels across age groups, which can be problematic due to differences in vascular reactivity (Samanez-Larkin and D'Esposito, 2008).

To correct for multiple comparisons in our ROI analyses, we applied a Bonferroni-Holm correction (Holm, 1979) across all ROIs for which we performed a particular analysis. All effects survived this correction unless otherwise indicated. Separate analysis types (e.g., main effects vs. correlations, and cue vs. word period) were treated as independent from each other for the purposes of this correction, as were the two age groups.

In order to characterize the temporal evolution of BOLD signal within individual ROIs, we ran a separate GLM analysis modeling the data 
Table 1

Mean (SE) proportion of items recalled on each list (including 2 practice lists), split by age group and value group.

\begin{tabular}{|c|c|c|c|c|c|c|c|c|}
\hline & & \multicolumn{7}{|l|}{ List } \\
\hline & & $\mathrm{P} 1$ & $\mathrm{P} 2$ & 1 & 2 & 3 & 4 & 5 \\
\hline \multirow[t]{2}{*}{ Young } & High & $0.500(0.056)$ & $0.675(0.048)$ & $0.646(0.059)$ & $0.693(0.039)$ & $0.713(0.045)$ & $0.804(0.035)$ & $0.763(0.044)$ \\
\hline & Low & $0.242(0.031)$ & $0.283(0.039)$ & $0.275(0.055)$ & $0.241(0.054)$ & $0.242(0.047)$ & $0.254(0.060)$ & $0.263(0.062)$ \\
\hline \multirow[t]{2}{*}{ Old } & High & $0.306(0.051)$ & $0.484(0.050)$ & $0.377(0.051)$ & $0.515(0.052)$ & $0.507(0.049)$ & $0.460(0.058)$ & $0.496(0.060)$ \\
\hline & Low & $0.234(0.041)$ & $0.258(0.052)$ & $0.145(0.030)$ & $0.178(0.046)$ & $0.178(0.043)$ & $0.170(0.052)$ & $0.159(0.041)$ \\
\hline
\end{tabular}

using finite impulse response (FIR) basis functions. For this model, we separated high-value and low-value trials, with each trial beginning at the time of cue onset. We modeled each trial as 10 single time points over a temporal window lasting $25 \mathrm{~s}$ from cue onset. Other parameters were the same as for the previously described GLM analysis. This FIR analysis generated separate parameter estimate maps for each $2.5 \mathrm{~s}$ peristimulus time bin for each condition, within a given run. For any given ROI, these parameter estimates could be averaged across voxels and runs, and then averaged across participants to yield group-level peristimulus time course plots.
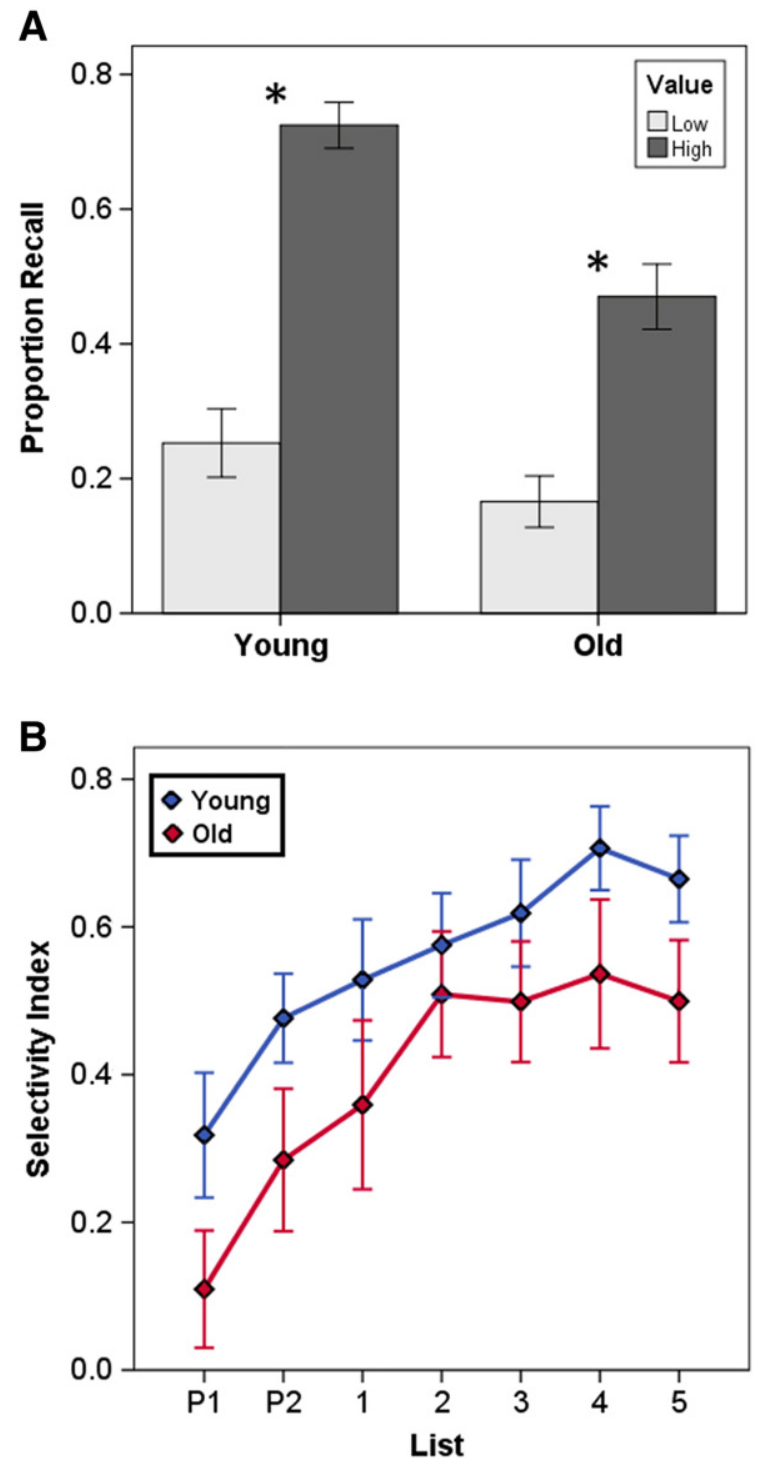

Fig. 1. (A) Mean proportion of items recalled per list, collapsed across all scanned lists. (B) Mean Selectivity Index as a function of list for young and older adults, including the two initial practice lists (P1 and P2) and the 5 scanned lists. Error bars represent \pm 1 SE.

\section{Results}

\section{Behavioral data}

We begin by examining how value affected the proportion of items recalled on the free recall tests in older adults (Table 1 ). ${ }^{2}$ A $2 \times 5$ (value $\times$ list) repeated measures ANOVA showed a highly reliable main effect of value (high vs. low), $F(1,20)=30.68$, MSE $=.160$, $p<.001, \eta_{\mathrm{p}}^{2}=.61$ (Fig. $1 \mathrm{~A}$ ). There was also a reliable main effect of list, $F(4,80)=3.64, M S E=.013, p=.009, \eta_{\mathrm{p}}^{2}=.15$, reflecting generally poorer performance on the first scanned list compared to later lists. The value $\times$ list interaction approached, but did not reach, significance, $F(4$, $80)=2.08, M S E=.012, p=.091, \eta_{\mathrm{p}}^{2}=.09$. Planned comparisons confirmed that high-value items were remembered better than low value items across each of the 5 scanned lists, all $t s>4.30, p<.001$. We also used separate paired-samples $t$-tests to examine value effects on the practice lists (Table 1 ). On the first practice list, there was not a reliable main effect of value, $t(20)=1.48, p=.154$, but the effect of value was reliable by the second practice list, $t(20)=3.26, p=.004$.

We also examined whether the proportion of items differed as a function of different point values within high-value and low-value groups in older adults, collapsing across lists (Table 2). A one-way repeated measures ANOVA examining only low-value items showed no effect of point value, $F(2,44)<1$. On high-value items, there was a trend towards an effect of value, but this effect did not reach significance, $F(2,44)=2.59, M S E=.010, p=.086, \eta_{\mathrm{p}}^{2}=.11$. In addition, the trend that was present was for better memory on 10-point items, not the advantage for 12-point items that might be expected if learners were showing sensitivity to points within the high-value group (Table 2). These results justify collapsing across values to form dichotomous high-value and low-value conditions (note that value levels were also dichotomized in Cohen et al., 2014).

As mentioned above, selectivity index provides a useful metric to quantify the impact of item value on memory recall performance. One-sample t-tests showed that selectivity index was significantly greater than zero across each of the 5 scanned lists in our sample of older adults (all ts $>3.14$, all $p s<.005$ ), and a one-way repeated measures ANOVA showed that selectivity index did not reliably change across these 5 lists, $F(4,76)=1.06, M S E=.071, p=.38, \eta_{\mathrm{p}}^{2}=.05$ (Fig. 1B). We also examined selectivity index on the two practice lists separately. On the first practice list, selectivity index was not reliably greater than zero, $t(18)=1.38, p=.184$, but on the second list it was greater than zero, $t(20)=2.95, p=.008 .^{3}$

Finally, we compared memory performance for young and older adults, collapsing items across lists. A $2 \times 2$ (value $\times$ age) mixed ANOVA with repeated measures on the first factor, on the proportion of items recalled, showed a main effect of age on memory, $F(1,41)=$ $11.65, M S E=.054, p=.001, \eta_{\mathrm{p}}^{2}=.22$, with older adults remembering

\footnotetext{
2 Two older adults and one young adult were excluded from the value $x$ list analysis that follows because we only had usable data for 4 out of 5 lists from these individuals, as noted above.

3 Note that selectivity index cannot be computed for a given list if zero items were recalled on that list, as was the case for the first practice list in two participants, and on at least one scanned list for two participants, which is why df varies for corresponding analyses.
} 
Table 2

Mean (and SE) proportion of items recalled across the five scanned lists by specific point value.

\begin{tabular}{|c|c|c|c|c|c|c|}
\hline & \multicolumn{3}{|l|}{ Low value } & \multicolumn{3}{|l|}{ High value } \\
\hline & 1 & 2 & 3 & 10 & 11 & 12 \\
\hline Young & $0.248(0.050)$ & $0.258(0.055)$ & $0.253(0.054)$ & $0.701(0.042)$ & $0.708(0.037)$ & $0.764(0.033)$ \\
\hline Old & $0.167(0.035)$ & $0.167(0.038)$ & $0.163(0.044)$ & $0.498(0.055)$ & $0.433(0.051)$ & $0.479(0.048)$ \\
\hline
\end{tabular}

fewer items than young adults (Fig. 1A). There was also a value $\times$ age interaction, $F(1,41)=5.34, M S E=.028, p=.026, \eta_{\mathrm{p}}^{2}=.12$, such that the effect of value on memory was weaker for older adults than for young adults (Fig. 1A). Simple effect analysis using independent samples ttests shows that older adults remembered fewer high-value items than young adults, $t(41)=4.18, p<.001$; older adults also recalled numerically fewer low-value items than young adults, but this effect was not statistically significant, $t(41)=1.39, p=.172$, possibly due to floor effects. When comparing the weighted average selectivity index across age groups in a separate analysis, there was a trend for older adults to have a somewhat lower selectivity index (Fig. 1B), but this difference was not statistically significant; correcting for unequal variances between groups, $t(38.39)=1.75, p=.088$.

In prior work using this type of task, older adults demonstrated equal or greater selectivity as young adults (cf., Castel et al., 2002, 2007, 2009; Castel, 2008). One reason why older adult participants in the present study may have instead shown a mild decrement in selectivity is that they had to perform the value-directed remembering task in the fMRI scanner with an interleaved vowel-consonant judgment baseline task. The vowel-consonant baseline may have functioned as a task-switching component within the encoding paradigm, and older adults tend to have difficulty with task switching (see Verhaeghen and Cerella, 2002, for a review). Nevertheless, as noted above, older adults showed a selectivity index that was robustly above chance across scanned blocks, regardless of any possible age-related decrement in selectivity. Thus, they still show a consistent preference for recall of high value words. Older adults' strong use of value here is broadly consistent with previous work using this task in which selectivity is retained or improved with healthy aging. Thus, we believe that it is inadvisable to overinterpret the possibility of a slight age-related reduction in selectivity; instead, we interpret our results in the context of older adults' remarkably preserved ability to selectively encode high-value items.

\section{Whole brain fMRI analyses}

\section{Word-encoding period}

First, we examined main effects of value across the entire brain in older adults during the word-encoding period (Fig. 2A; Supplemental Table 1A). This analysis revealed a number of areas with greater activity during encoding of high-value words than during encoding of lowvalue words, including ventral and posterior portions of the left PFC and areas of left lateral temporal, left parietal, and bilateral occipital cortex. In addition, one cluster within the angular gyrus was less active during encoding of high-value words (Fig. 2A; Supplemental Table 1B), presumably reflecting default-mode network deactivation (e.g., Raichle et al., 2001), or something akin to what have previously been termed "negative subsequent memory" effects (e.g., Otten and Rugg, 2001; Daselaar et al., 2004; Mattson et al., 2015).

When comparing these effects to the analogous contrast in young adults, we find considerable overlap across the two age groups, including in the ventral and posterior regions of left lateral PFC (Fig. 2B; Supplemental Table 2A). However, despite these commonalities, there were some notable differences. One theoretically important difference is that, while young adults show reliable effects of value in right lateral PFC, older adults do not. Theories of reduced hemispheric asymmetry in older adults (e.g., Cabeza, 2002) would make the opposite prediction.
That is, Cabeza's HAROLD model would predict that older adults should show a more bilateral pattern of activity than young adults, but in fact, older adults seem to show an even stronger left-lateralization than do young adults in this task. Thus, our data do not support the idea that older adults compensate for neural deficits by engaging the hemisphere contralateral to the one in which a task is typically performed.

Other age-related differences are also apparent. Specifically, the spatial extent of value-related activity modulations is generally less diffuse in older adults. For example, young adults show value effects in caudate nucleus that are not found in older adults. Additionally, we find statistically reliable age differences in the degree to which activity in left IFG, left superior temporal gyrus, and bilateral pre-supplementary motor area (pre-SMA) are modulated by value. Even in some portions of left IFG that show value effects in both age groups, there are regions in which those effects are reliably stronger for young adults. Finally, one cluster near the parietal/occipital junction (precuneus/cuneus) shows greater deactivation on high-value items relative to low-value items in young adults relative to older adults (Supplemental Table 2B). This age-related difference may correspond to age-related reductions in default-mode network deactivations (cf. Persson et al., 2007), and to age-related attenuation and/or reversal of negative subsequent memory effects (cf., Duverne et al., 2009; Mattson et al., 2015). No other regions showed age-related differences in value effects; in other words, there were no regions for which older adults showed a stronger main effect of value than did young adults.

Exploring main effects of value is a start for understanding how encoding-related activity is modulated by value. However, this analysis does not tell us which value-related differences in activity at encoding actually lead to more selective memory for valuable items. One approach to addressing that important question would be to compare encoding-related activity for subsequently-recalled vs. subsequentlyforgotten items, separated by value. However, such an analysis turns out to be problematic in the present study, as many participants had an insufficient number of high-value forgotten items and/or an insufficient number of low-value remembered items. Older adults were particularly unlikely to remember a sufficient number of low-value words to allow us to generate reliable estimates of brain activity for those items. We thus pursued an alterative analysis approach focused on individual differences. Specifically, we computed the across-subjects correlation between value-related differences in brain activity and participants' selectivity indices. That is, we look for regions in which greater value-related differences in brain activity during encoding are associated with stronger behavioral effects of value on subsequent memory. Although we feel that this is a superior analysis strategy given the constraints inherent in our data set, for completeness sake we also performed a standard subsequent memory analysis on the data from only those individuals who had sufficient trials of each type to enable us to do so; those analyses are discussed further in the Supplemental Results.

In young adults, we previously reported a correlation between selectivity index and value-related modulation of activity in a largely leftlateralized network of prefrontal and temporal regions (Cohen et al., 2014). The next question, then, is whether older adults show a similar pattern of results. We indeed find that in older adults, there is a leftlateralized network of regions for which value-related differences in activity correlate with selectivity index (Fig. 3A; Table 3). Specifically, we find clusters consistent with this pattern in $\mathrm{L}$ ventral and posterior 

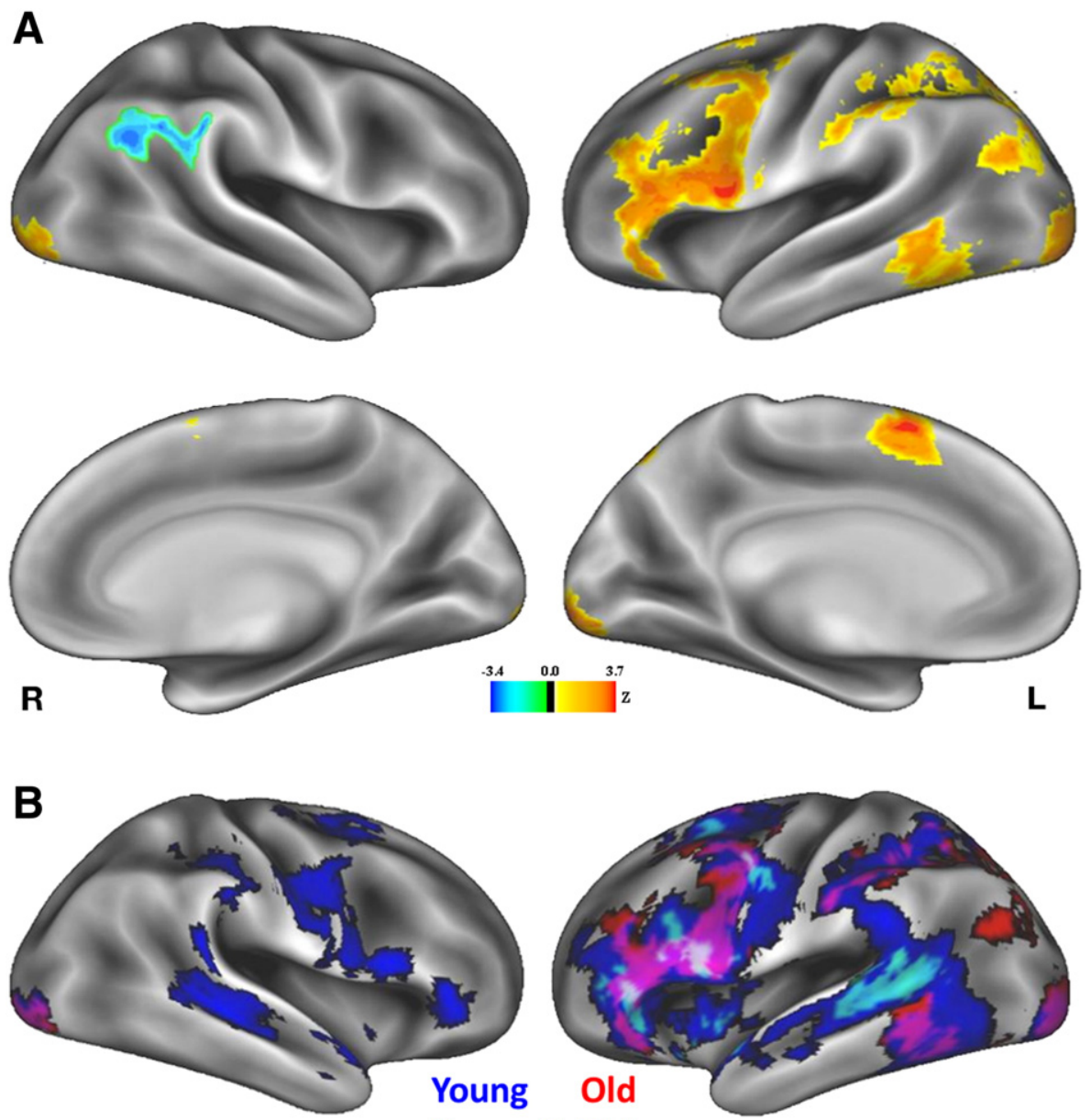

Young \& Old
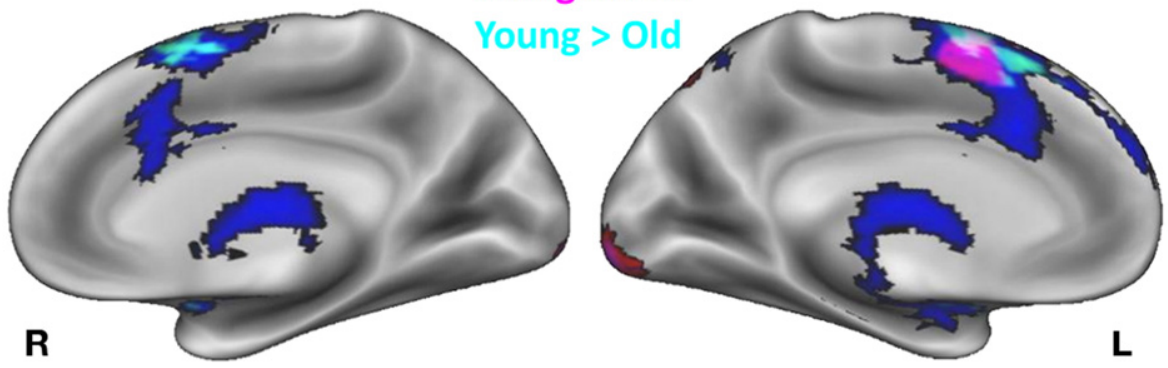

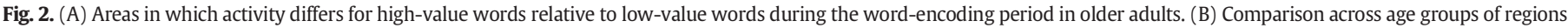

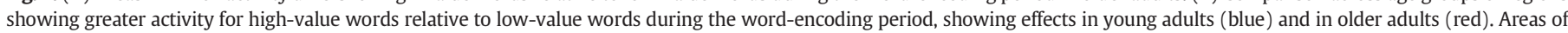

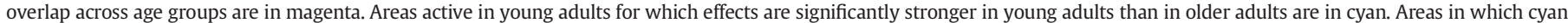
and magenta colors overlap are in white.

lateral PFC, L posterior lateral temporal cortex, and L pre-SMA. Thus, there is evidence that value-related modulation of semantic processing is associated with the degree of memory selectivity in both age groups.

When we compare these results directly with the analogous brainbehavior correlation analysis in young adults (Supplemental Table 3), we do see some subtle but potentially important differences (Fig. 3B; Table 4). One area in which the correlation with selectivity is significantly stronger in young adults than in older adults is in the most anterior portion of pre-SMA (colored in cyan in Fig. 3B). At the same time, more posteriorly in the SMA, there is an effect in older adults that is not reliably present in young adults, although here there is no reliable age difference. A cluster in the most ventral portion of the left IFG also shows a significant interaction with age, with young adults but not older adults showing a reliable correlation between selectivity index and the degree of value-related activity modulation. Additionally, the left prefrontal regions that showed the maximal correlation effects appear to be shifted in a dorsal and posterior direction in older adults as compared to young adults, although there is not a statistically significant age difference other than in the small ventral IFG cluster noted above.

\section{Separation of word period effects by condition}

It is also worth considering whether the relationship between valuerelated differences in activity and memory selectivity is being driven by high-value items, low-value items, or both, across the two age groups. Indeed, this analysis suggests another age-related difference. Specifically, in young adults, there are a number of brain regions in which increased activity during encoding of high-value items, relative to baseline, correlates positively with selectivity index (Fig. 4A; Supplemental Table 4A). This analysis reveals many of the same left inferior prefrontal regions that are apparent in Fig. 3, as well as some activity in the anterior temporal lobe and insula. In contrast, a similar analysis looking 

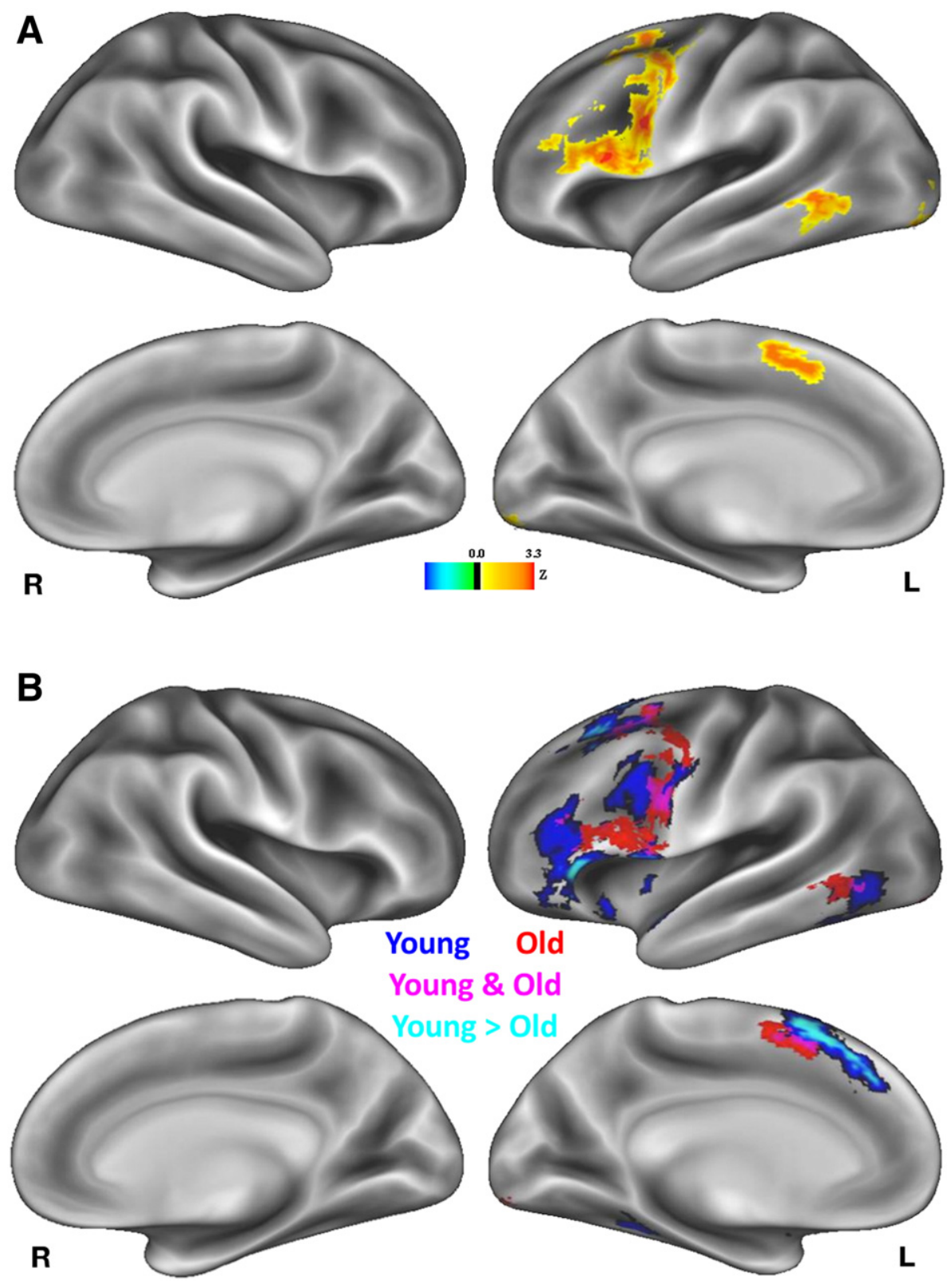

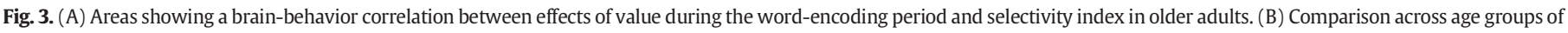

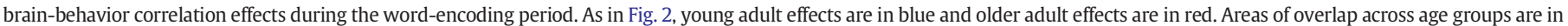
magenta, while areas active in young adults for which effects are significantly stronger in young adults than in older adults are in cyan.

only at the relationship between low-value item encoding activity and selectivity index does not reveal any significant effects. If we extract the voxels displayed in Fig. $4 \mathrm{~A}$ as an ROI, we find that in our young adult sample, activity in this region during encoding of high-value items is significantly greater than activity during encoding of lowvalue items, $t(19)=4.02, p<.001$, and the magnitude of valuerelated difference in activity correlates positively with selectivity index, $r=.65, p=.002$ (note that the dependent measures used in these ROI-based analyses are distinct from that used in the ROIdefining contrast, thus avoiding potential concerns of statistical circularity). Thus, it seems likely that in young adults, the brain-behavior correlation shown in Fig. 3B is being driven largely by effects on high-value items.

In older adults, the pattern is different. There is an apparent network of regions for which activity during encoding of high-value items correlates positively with selectivity index (Supplemental Fig. 1; Supplemental Table 4B); however, this network does not seem to overlap with the regions shown in Fig. 3. Instead, the effects are largely right-lateralized, including $\mathrm{R}$ anterior temporal cortex and $\mathrm{R}$ angular gyrus, as well as bilateral ventromedial prefrontal cortex. Extracting this network as an $\mathrm{ROI}$, it is clear that activity in these regions is not greater during encoding of high-value words; indeed, if anything, the trend across these regions is for numerically greater activity during encoding of low-value items, $t(22)=1.43, p=.166$. In addition, while there is a trend for a positive correlation between the value-related differences in activity and selectivity index, that trend is not significant, $r=.33$, $p=.123$. Thus, whatever the meaning of individual differences in activity during high-value items in older adults, it seems unlikely that they underlie the key effects shown in Fig. 3.

In older adults, examining activity during encoding of low-value items proves more informative. Fig. 4B (see also Supplemental Table 4C) demonstrates a largely left-lateralized network showing a negative correlation with selectivity, which includes posterior L ventrolateral prefrontal cortex, L posterior inferior temporal cortex, and 
Table 3

Activation peaks for regions in which high-low difference in brain activity during the word-encoding period correlates with selectivity index for older adults.

\begin{tabular}{|c|c|c|c|c|c|c|c|}
\hline Cluster & Peak & $\mathrm{BA}$ & z-stat & $\mathrm{X}$ & $\mathrm{Y}$ & Z & Voxels \\
\hline \multirow[t]{5}{*}{1} & $\begin{array}{l}\text { L inferior frontal gyrus, } \\
\text { pars opercularis }\end{array}$ & 44 & 3.67 & -48 & 12 & 28 & 1445 \\
\hline & L precentral gyrus & 6 & 3.41 & -44 & -2 & 54 & \\
\hline & $\begin{array}{l}\text { L middle/superior frontal } \\
\text { gyrus }\end{array}$ & 6 & 3.04 & -30 & 16 & 56 & \\
\hline & $\begin{array}{l}\text { L inferior frontal gryus, } \\
\text { pars triangularis }\end{array}$ & 45 & 3.00 & -48 & 28 & 22 & \\
\hline & L middle frontal gyrus & 9 & 2.56 & -52 & 22 & 32 & \\
\hline 2 & R cerebellum (posterior) & - & 3.54 & 32 & -70 & -30 & 397 \\
\hline \multirow[t]{3}{*}{3} & L lingual gyrus & 17 & 4.22 & -14 & -108 & -8 & 381 \\
\hline & L inferior occipital gyrus & 18 & 3.57 & -36 & -96 & -6 & \\
\hline & L fusiform gyrus & 19 & 3.04 & -26 & -86 & -14 & \\
\hline \multirow[t]{2}{*}{4} & $\mathrm{R}$ lingual gyrus & $17 / 18$ & 5.06 & 24 & -102 & 0 & 376 \\
\hline & $\mathrm{R}$ inferior occipital gyrus & 18 & 3.35 & 28 & -88 & -4 & \\
\hline \multirow[t]{2}{*}{5} & $\begin{array}{l}\text { L middle/inferior temporal } \\
\text { gyrus }\end{array}$ & 37 & 3.62 & -60 & -56 & -8 & 363 \\
\hline & L inferior occipital gyrus & 19 & 3.33 & -56 & -68 & -2 & \\
\hline \multirow[t]{3}{*}{6} & $\begin{array}{l}\text { L medial frontal gyrus } \\
\text { (supplementary motor } \\
\text { area) }\end{array}$ & 6 & 3.26 & -4 & 2 & 56 & 312 \\
\hline & L paracingulate gyrus & 32 & 3.03 & -2 & 12 & 46 & \\
\hline & L superior frontal gyrus & 6 & 2.92 & 0 & 12 & 56 & \\
\hline
\end{tabular}

L pre-SMA, as well as L hippocampus. These regions seem to largely match the areas showing a brain-behavior correlation in older adults in Fig. 3. Indeed, when extracting this network as an ROI, we find significantly greater activity during encoding of high-value relative to low-value items in older adults in this network, $t(22)=3.63, p=$ .001 , and a positive correlation between those differences and selectivity index, $r=.67, p<.001$. Thus, it seems likely that in older adults, the correlation between value-related differences in brain activity and selectivity index is being driven by reduced activity during encoding of low-value items in regions in which activity typically benefits encoding.

Fig. 4B also shows a largely right-lateralized network in which activity during encoding of low-value items correlates positively with selectivity index (see also Supplemental Table 4D). The regions showing such effects include $\mathrm{R}$ angular gyrus, $\mathrm{R}$ posterior cingulate, and $\mathrm{R}$ precuneus, and $R$ ventromedial prefrontal, all areas that would seem to reflect default-mode network activity. When we extract this network as an ROI, we find greater activity during encoding of low-value relative to high-value items in these regions in older adults, $t(22)=3.28, p=$ .003 , although there is not a significant correlation between valuerelated differences in activity and selectivity index across this network, $r=-.28, p=.199$. It may be that activation of these regions during low-value items is indicative of participants turning their attention inward, away from the low-value stimuli that are on screen, and that this process benefits overall selectivity.

\section{Cue period}

In young adults, we previously identified a number of regions exhibiting increased activity during the cue period of the trial when the cue signaled that the upcoming item would have high value, versus low value, if later remembered (Cohen et al., 2014). These cue-period

\section{Table 4}

Activation peaks for regions in which Young > Old for the correlation between the High Low value difference in activity and selectivity index, masked by voxels showing this effect in either young or older adults.

\begin{tabular}{llllllll}
\hline Cluster & Peak & BA & Z-Stat & X & Y & Z & Voxels \\
\hline 1 & L superior frontal gyrus & 6 & 3.43 & -2 & 22 & 58 & 388 \\
2 & L inferior frontal gyrus, & $45 / 47$ & 3.44 & -50 & 26 & -4 & 162 \\
& $\begin{array}{l}\text { pars triangularis } \\
\text { L inferior frontal gyrus, }\end{array}$ & 44 & 3.27 & -52 & 14 & -2 & \\
& pars opercularis & & & & & & \\
& & & & & &
\end{tabular}

value effects-observed in high-level control areas such as left lateral PFC and reward-sensitive regions such as nucleus accumbensoverlapped somewhat with the network of regions showing main effects of value during the word-encoding period. Interestingly, a whole brain analysis conducted on our present sample of older adults revealed no main effects of value during the cue period; i.e., no regions showed significantly greater or lesser activity in response to high value cues. When directly comparing activity between young adults and older adults for this contrast, there were prefrontal, parietal, and occipital clusters that emerged as showing a reliable value $\times$ age interaction, such that the effects of value were larger in young adults than in older adults (Fig. 5; Supplemental Table 5). Thus, it seems clear that older adults are much less responsive to value during the cue period than are young adults. These age differences may reflect broader age differences in the deployment of pre-stimulus encoding-related processes.

We next ran whole-brain analyses searching for any brain regions in which value-related differences in activity during the cue period were associated with individual differences in selectivity index. In neither the young nor the older adult groups did we find any reliable clusters showing correlations between value-related activity differences and memory selectivity during the value cue period, before the to-beremembered word was presented. If we assume that our acrossparticipants brain-behavior correlation analysis is roughly analogous to a more typical within-participants subsequent memory analysis, our findings differ from many earlier studies (e.g., Adcock et al., 2006; Park and Rugg, 2010; Bollinger et al., 2010; Addante et al., 2015). Those studies emphasized how, in young adults, a stronger brain response to a pre-stimulus cue is associated with better subsequent memory for the item that appears immediately after that cue. Instead, in our paradigm, value-related differences in the brain response to the cue appear to be inconsequential for later memory in both age groups. We thus conclude that pre-stimulus preparatory activity, and any agerelated differences in the deployment of these mechanisms, is less consequential in the present paradigm than in the tasks used in prior studies (cf., Bollinger et al., 2011).

\section{Region of interest analyses}

In order to better understand the patterns of effects seen in the whole brain analyses, we also performed ROI analyses to investigate the engagement of networks supporting semantic processing and reward during performance on this task. Our ROI analysis involved clusters of regions gathered from automated meta-analyses using the Neurosynth database (http://neurosynth.org; Yarkoni et al., 2011). We generated "reverse inference" maps from the database for the terms "semantic" and "reward". We chose to examine the "semantic" network based on our hypothesis that preferential engagement of deep semantic processing is one important strategic mechanism for bolstering the memorability of high value words. This allowed us to define an ROI for further analyses that is not statistically dependent on our whole-brain results. We also generated a "reward" network ROI, in order to facilitate comparisons with prior relevant studies that focused on such a mechanism (e.g., Adcock et al., 2006). Note that in both cases, we use the term "ROI" not to refer to a single focal region of interest but rather to a larger mask that encompasses several different brain areas.

The initial maps generated by the Neurosynth software included voxels that were more likely to be activated in the studies with a given index term than would be expected by chance, with an FDRcorrected threshold of $p<.01$. Out of 9721 total studies in the database, 701 were indexed with the term "semantic" and 497 were indexed with the term "reward". To ensure that the resulting ROIs were not excessively diffuse in their spatial extent, we applied an additional voxelwise threshold of $z>5.20$, producing an alpha level of $p<.0000001$, onetailed. The "semantic" ROI included 4501 voxels, and produced a network that included large areas of $\mathrm{L}$ inferior frontal gyrus and $\mathrm{L}$ lateral temporal cortex (Fig. 6A). The "reward" ROI included 3827 voxels, and 

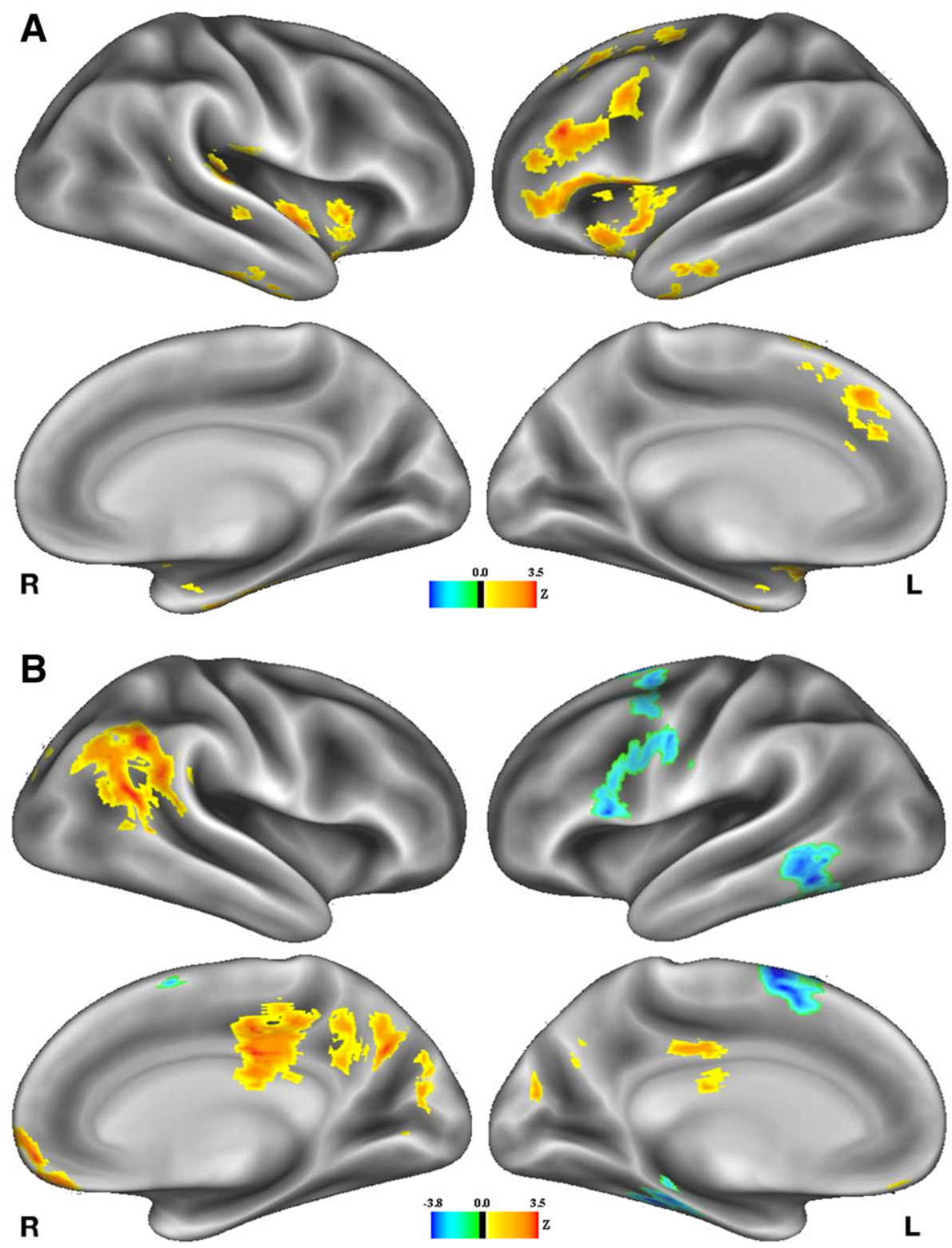

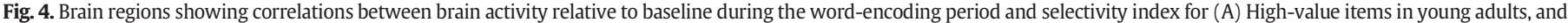

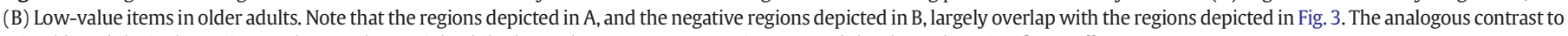
A in older adults is shown in Supplemental Material, while the analogous contrast to B in young adults showed no significant effects.

produced a network including bilateral nucleus accumbens, ventral tegmental area, and ventromedial PFC (Fig. 9A).

\section{Neurosynth semantic ROI}

We first examined how activity across the Neurosynth-derived semantic network ROI is associated with main effects of value, and how these value effects correlate with selectivity index. During the wordencoding period, activity within the semantic network ROI was reliably greater for high-value items, both in young adults, $t(19)=4.94, p<.001$ (Fig. 6B), and in older adults, $t(22)=3.55, p=.002$ (Fig. 6C). A $2 \times 2$ (value $\times$ age) mixed ANOVA with repeated measures on the first factor finds that the interaction between these factors approaches significance, $F(1,41)=4.05, M S E=107.02, p=.051, \eta_{\mathrm{p}}^{2}=.09$, suggesting that the effect may have been somewhat stronger in young adults. We also examined how value-induced modulation of activity in this semantic network ROI relates to selectivity index. The magnitude of value-related changes in brain activity in the semantic ROI during the wordencoding period correlated with selectivity index in young adults, $r=$ $.54, p=.015$ (Fig. 6D), as well as in older adults, $r=.57, p=.005$
(Fig. 6E). These findings suggest that in both age groups, the magnitude of value-related differences in the degree to which a given individual activates semantic processing areas of the brain during word encoding correlates with how selective they are as a function of value at the time of the recall test.

Consistent with the whole-brain results described above, we do, however, see interesting age-related differences when examining how encoding activity for high and low value words, relative to baseline, is differentially related to subsequent selectivity. In young adults, activity during encoding of high-value words correlated positively with selectivity, $r=.52, p=.018$, while activity during encoding of low-value words was uncorrelated with selectivity, $r=.08, p=.736$ (Fig. 7A). We compared the two correlation coefficients via a test of dependent correlation coefficients (Steiger, 1980; Revelle, 2015), and found that the difference is significant, $t(18)=2.60, p=.018$. In older adults, activity during encoding of high-value words was uncorrelated with selectivity index, $r=.04, p=.860$, but activity during encoding of low-value words was significantly negatively correlated with selectivity index, $r=-.48, p=.019$ (Fig. 7B). The difference between the 

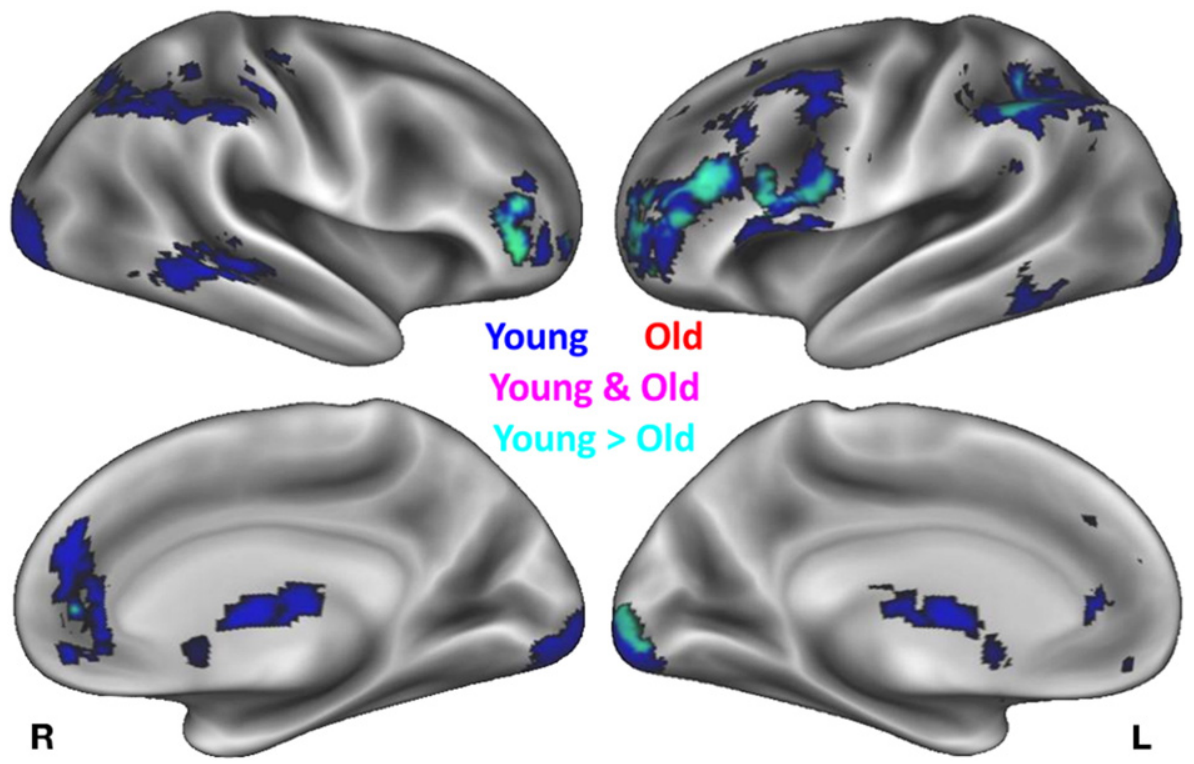

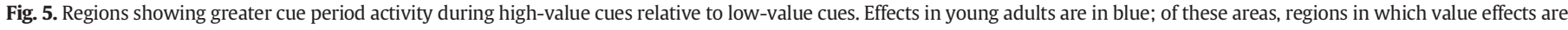
significantly greater in young adults than in older adults are in cyan. No areas showed value effects during the cue period in older adults.

correlations is significant, $t(21)=4.69, p<.001$. Thus, it seems that in young adults, selective recall is associated with stronger activation of semantic-processing regions, relative to baseline, during encoding of high-value words, while in older adults, selective recall is associated with reduced activation of semantic processing regions, relative to baseline, during encoding of low-value words.

Another important age difference emerged during the cue period. Young adults showed greater activity for high-value cues, $t(19)=$ 2.97, $p=.008$ (Fig. 6B), while older adults showed no effect of value on cue period activity, $t(22)<1$ (Fig. $6 C$ ). We also found a reliable value $\times$ age interaction during the cue period, $F(1,41)=6.67$, MSE $=$ $149.75, p=.013, \eta_{\mathrm{p}}^{2}=.14$, confirming that young adults show a significantly stronger effect of value than older adults. Cue period activity did not correlate with selectivity index in young adults, $r=-.03, p=.888$ (Fig. 6F), nor did cue period activity correlate with selectivity index in older adults, $r=.19, p=.392$ (Fig. 6G). Thus, there were striking age differences in how value affected brain activity during the cue period, consistent with age-related differences in proactive responding to the value cue. Still, despite the fact that value-related differences in activity across this same network during the word-encoding period strongly correlated with memory selectivity, value-related differences in activation during the cue period did not appear to contribute to this selectivity.

In addition to the standard GLM analysis, we used a finite impulse response (FIR) model to generate peristimulus time course plots of BOLD signal averaged across the semantic network ROI. This analysis makes no a priori assumptions about the shape and timing of the BOLD signal associated with component stages of the task. Thus, it allows for a stronger test of our contention that the apparent age differences in how value affected brain activity during different stages of the trial (cue period vs. word period) reflect a true difference in older adults' cognitive responses, rather than, for example, a generally slowed HRF in older adults. Visual inspection of Fig. 8A finds a value-related difference in activity early in the trial, 2.5-7.5 s after cue onset, in young adults, which likely corresponds to the brain response to the cue. Older adults do not appear to show a differential response during that same time period (Fig. 8B). Later in the trial, however, 10-12.5 s after cue onset, it is apparent that both young adults and older adults show a notably stronger BOLD signal during high-value trials, compared to low-value trials. We can assume that this change in BOLD signal is in response to the appearance of the word on the screen, which occurs between 5 and $8.75 \mathrm{~s}$ after cue onset. From $15 \mathrm{~s}$ to $22.5 \mathrm{~s}$ after cue onset, the last part of the modeled time window, both brain activity and the value-related difference in activity is greatly reduced in both age groups. It appears that by this point in the trial, encoding-related activity for the newly-presented item is largely complete, with no agerelated slowing of the BOLD response visible in older adults.

Paired-samples t-tests on the mean parameter estimates within each time interval, with a Bonferroni-Holm correction for multiple comparisons applied for the four tested time intervals within each age group, confirm the above description of the results. There was no value-related difference in activity at the first time point $(0 \mathrm{~s})$ for either age group: $t(19)<1$ in young adults, and $t(22)=1.36, p=.19$ in older adults. In young adults, there was a significant value-related difference in activity across the average of parameter estimates from the next three time points (2.5s-7.5 s), $t(19)=3.49, p=.002$, while we did not find any such effects in older adults, $t(22)<1$. A $2 \times 2$ (value $\times$ age) mixed ANOVA, with repeated measures on the first factor, confirms a value $\times$ age interaction, $F(1,41)=7.09, M S E=.015, p=.011, \eta_{\mathrm{p}}^{2}=$ .15 . Thus, the FIR analysis replicates the results from our standard model-based GLM analysis, indicating that young adults show effects of value during the cue period while older adults do not.

We next interrogated FIR activity estimates obtained from time points 5 (10 s) and 6 (12.5 s), which presumably reflect the brain response to the appearance of the to-be-remembered word stimuli, based on the large increase in activity relative to baseline. When parameter estimates are averaged for these two time points, young adults show a significant effect of value on BOLD signal, $t(19)=4.83$, $p \leq .001$, and older adults also show a significant effect of value on BOLD signal, $t(22)=3.58, p=.002$. A $2 \times 2$ (value $\times$ age) mixed ANOVA on the average parameter estimates shows that the value $x$ age interaction is not significant, $F(1,41)=2.55$, MSE $=.026, p=$ $.118, \eta_{\mathrm{p}}^{2}=.06$. Thus, both young adults and older adults show more activity during high-value items than during low-value items when responding to the to-be-encoded word, again replicating the results from our GLM analysis.

Finally, we examine how value affected brain activity towards the end of each trial, 15-22.5 s after cue onset. This period is critical to rule out the alternate explanation that the HRF response to value is generally slowed in older adults. Averaging parameter estimates across the final four time points, young adults did not show a significant value effect, $t(19)=1.74, p=.098$, and older adults also did not show a 
A
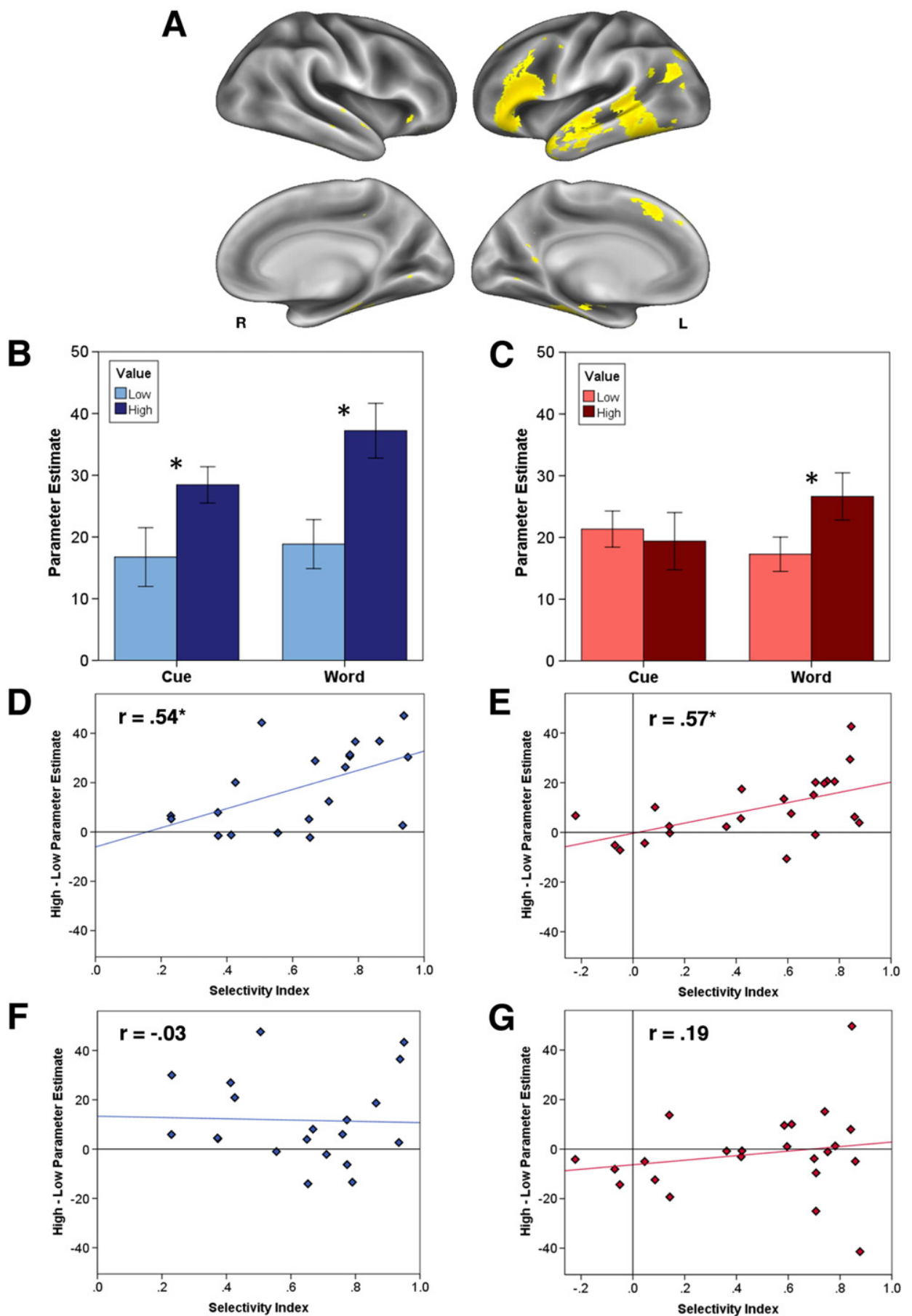

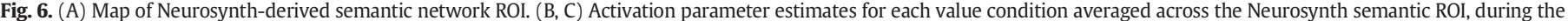

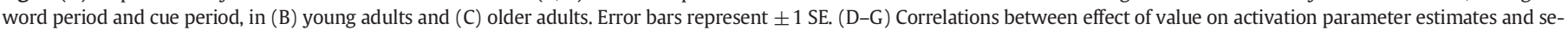
lectivity index. (D) Young adults, word period; (E) Older adults, word period; (F) Young adults, cue period; (G) Older adults, cue period.

significant effect of value, $t(22)<1$. A $2 \times 2$ (value $\times$ age) mixed ANOVA on the parameter estimates averaged across these final four time points found a marginally significant value $\times$ age interaction, $F(1,41)=3.87$, $M S E=.012, p=.056, \eta_{\mathrm{p}}^{2}=.09$. However, the trend, if anything, is for stronger value effects in young adults, contrary to the idea of a general slowing of the hemodynamic response in older adults. Therefore, this potential alternate explanation for the observed age-related difference in the temporal extent of responsiveness to value is unlikely to explain our findings. Instead, it seems clear that the effect of value is more temporally limited in older adults than in young adults, supporting our conclusion that older adults only show differential brain activity as a function of value while the word is on-screen, and not in response to the cue.

\section{Neurosynth reward ROI}

We also examined how value affects activity in reward-sensitive regions, specifically using the Neurosynth-derived reward ROI (Fig. 9A). During the word-encoding period, we found that young adults showed a main effect of value, $t(19)=4.14, p=.001$ (Fig. 9B), while older adults did not, $t(22)=1.45, p=.16$ (Fig. 9C). There was a trend for a value $\times$ age interaction during the word period, $F(1,41)=4.42$, $M S E=32.17, p=.042, \eta_{\mathrm{p}}^{2}=.10$, but the effect does not surpass the multiple-comparison-corrected $\alpha$ of .025 . Additionally, young adults showed a correlation between selectivity index and effect of value in the reward ROI during the word period, $r=.54, p=.013$, while older adults did not, $r=.21, p=.344$. 

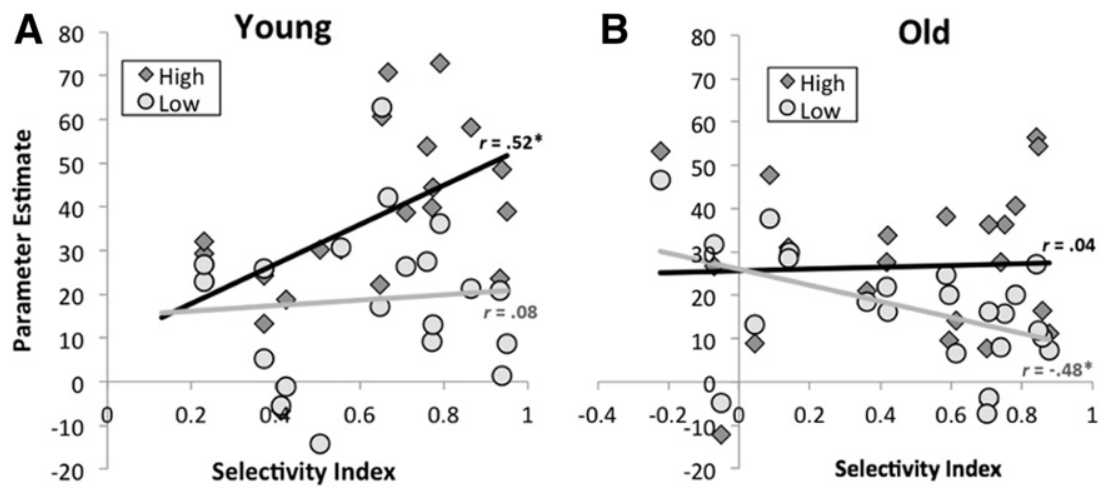

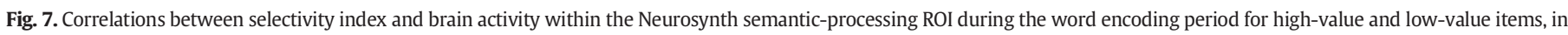
(A) young adults and (B) older adults.

Young adults also showed a main effect of value during the cue period, $t(19)=3.15, p=.005$ (Fig. 9B), while older adults did not, $t(22)<1$ (Fig. 9C); here, the value $\times$ age interaction was significant, $F(1,41)=$ $6.90, M S E=111.78, p=.012, \eta_{\mathrm{p}}^{2}=.14$. However, in the cue period, there was no correlation between selectivity index and the effect of value in the reward network ROI in young adults, $r=-.09, p=.699$, nor in older adults, $r=.11, p=.616$. Young adults therefore reliably show greater activity in reward-sensitive regions during both highvalue words and during the cues that precede high-value words, relative to low-value words. These value-related differences in activity seem to affect selectivity in later recall when they happen during word encoding, but not when they precede encoding. Older adults do not show any sensitivity to value in reward-sensitive regions, suggesting that their performance on this task may be less driven by anticipation of potential future rewards than is the case for young adults.

\section{Discussion}

When faced with a daunting number of words to memorize, only some of which are deemed to be highly valuable, both younger and older adults can effectively calibrate their encoding strategy to prioritize these important items. Our fMRI results showcase how individuals of both age groups achieve this selectivity by regulating activity across a common set of left-lateralized brain regions based on the value of the words. These brain areas, which include VLPFC, posterior dorsal medial $\mathrm{PFC} /$ pre-supplementary motor area (pre-SMA), and posterior lateral temporal cortex, have all been associated with deep semantic processing (Binder et al., 2009; Binder and Desai, 2011). Specifically, value-related differences in activity in these brain regions during the word-encoding period correlate with selectivity index on the later recall test. We also observe a similar pattern of effects across a semantic processing network ROI derived from the Neurosynth meta-analysis database. We acknowledge that it is impossible to directly infer psychological processes from brain activity, and we did not collect self-report measures that were sufficient to produce a clear narrative description of the strategies for which modulation by value affects memory. Still, the profile of neural activity putatively suggests that, similar to young adults (Cohen et al., 2014), older adults are strategically controlling the degree to which they engage semantic processing during encoding of high-value items relative to the degree of such engagement during encoding of low-value items. In other words, the relative preservation of memory selectivity across the lifespan is accompanied by a general preservation in control of this semantic processing circuit.

It is notable, however, that higher selectivity indices in young adults seem to be driven largely by the degree of increased activity in semantic processing regions during encoding of high-value words, while selectivity in older adults seems to be tied more closely to what happens during encoding of low-value words. Specifically, selectivity indices in older adults seem to be higher based on how much an individual refrains from activating regions related to semantic processing during encoding of low-value words. Thus, there does appear to be an age-related difference in how, precisely, value-related modulation of activity in semantic processing regions affects later recall.

\section{Effects during word encoding}

One important piece of the semantic processing circuit that is differentially engaged depending on stimulus value is in left VLPFC, also described as left IFG. In a number of prior studies (e.g., Wagner et al.,
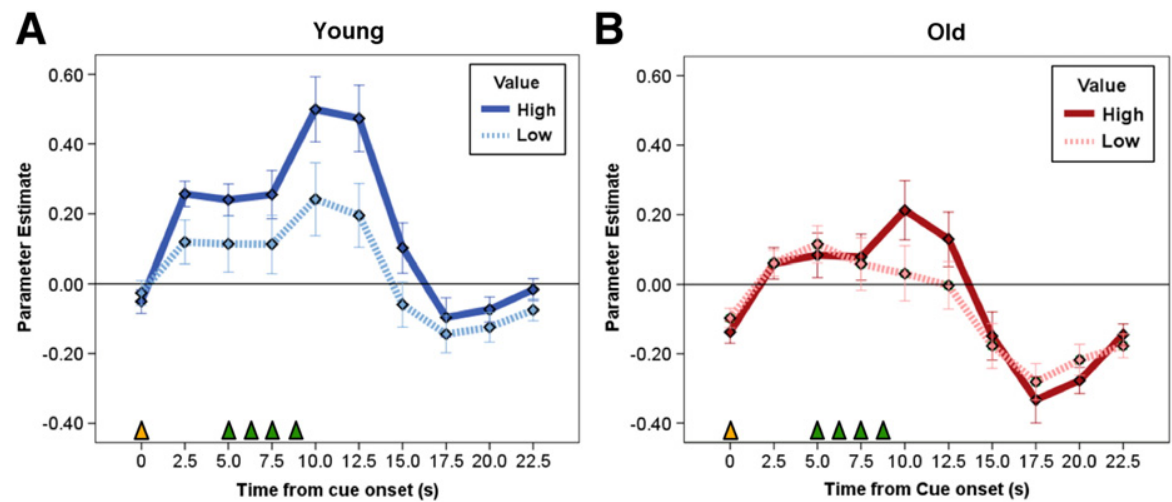

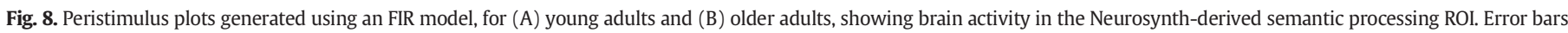

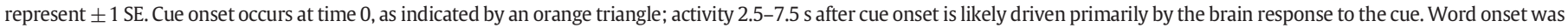

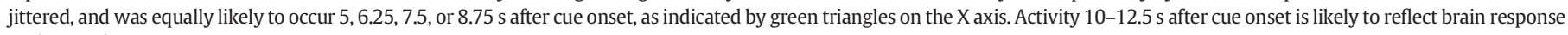
to the word. 

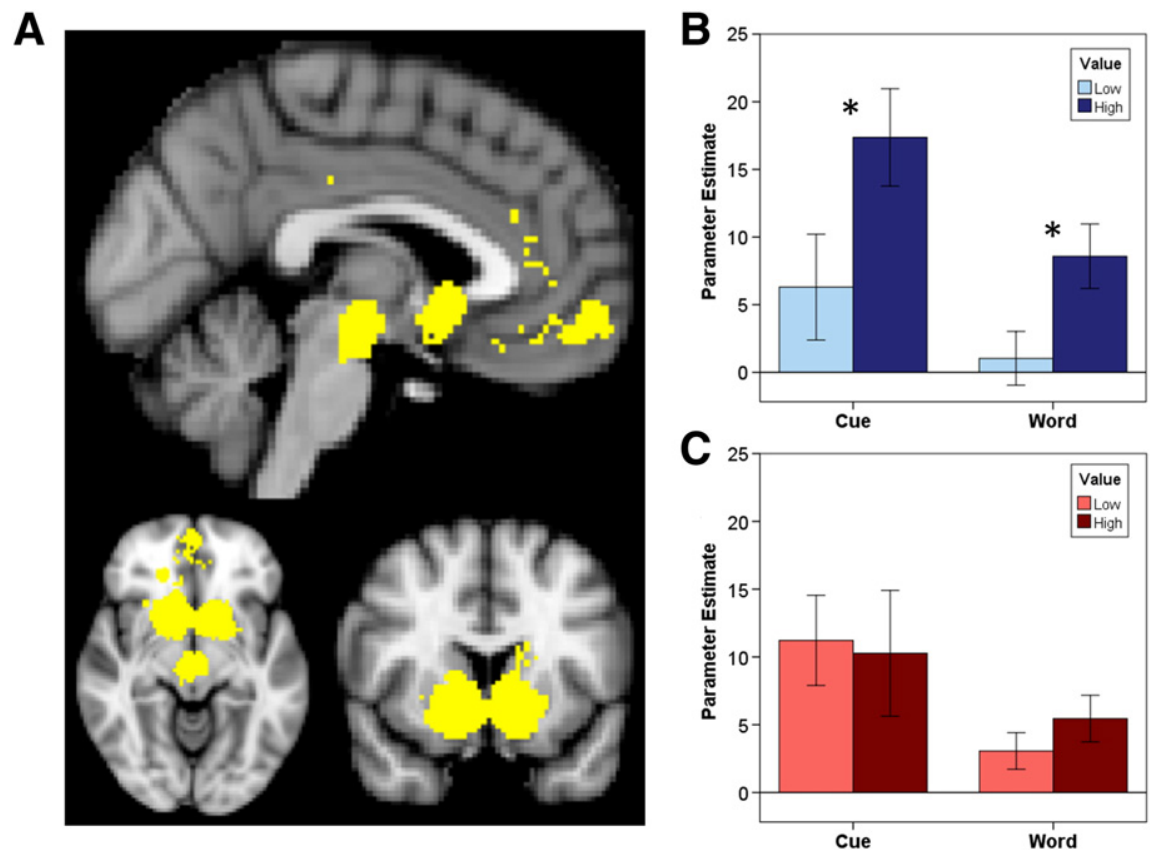

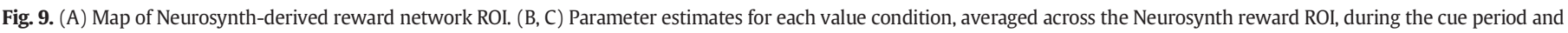
word period, for (B) young adults and (C) older adults. Error bars represent $\pm 1 \mathrm{SE}$.

1998; Savage et al., 2001; Paller and Wagner, 2002; Addis and McAndrews, 2006; Kirchhoff and Buckner, 2006; Miotto et al., 2006), activation of left inferior prefrontal regions during encoding in young adults has been associated with the use of effective semantic strategies, as well as with a concomitant increase in performance on a subsequent memory test. More recent work (Kirchhoff et al., 2012; Miotto et al., 2014) has shown that older adults who are trained to engage semantic encoding strategies also show increased activity in left IFG, among other regions, during encoding. It is notable that when we compare the six activation peaks in left IFG from across those two studies (transformed from Talairach to MNI space as necessary; Lancaster et al., 2007) with our whole-brain analysis examining the correlation between value effects during encoding and selectivity index (see Fig. 3A), we find that four of the six peaks from these prior studies overlap with significant clusters in our data, and the other two peaks are only one voxel away. Thus, although the critical left IFG effects in the mapwise brainbehavior correlation analysis do not overlap precisely across age groups, there is still solid evidence to suggest that left IFG effects in older adults reflect strategic engagement of semantic processing, similar to what we found previously for young adults (Cohen et al., 2014).

Value-related activity differences in two other areas also show correlations with selectivity index in older adults: posterior lateral temporal cortex, including middle temporal gyrus (MTG), and pre-SMA. Prior work (e.g., Whitney et al., 2011; Wagner et al., 2001) has suggested that processing in both left IFG and left posterior MTG is required for tasks that involve retrieval of semantic information, particularly when the semantic associations are weak, and thus more difficult. The strong influence of pre-SMA in both age groups is also notable. Prior studies have shown that this area is specifically involved in relatively unconstrained, internally-directed semantic generation tasks. For instance, Crosson et al. (2001) found that activity in pre-SMA was more broadly apparent with self-paced covert generation of category members and less apparent when generation was experimenter-paced. Activity was further reduced when cues guided the specific exemplars that were to be produced. Similarly, Tremblay and Gracco (2006) found greater activation in pre-SMA as well as L IFG when participants were asked to generate a word from a broader relative to a narrower category space. Thus, all three of the regions in which value-related modulation of activity during word encoding correlates with selectivity in older adults are relevant to the use of self-generated semantic strategies.

Gazzaley and colleagues have found that older adults have trouble inhibiting processing of irrelevant stimuli, and that the degree to which individual older adults are able to suppress neural responses to irrelevant stimuli correlates with better short-term memory for the to-be-attended stimuli (Gazzaley et al., 2005; see also Gazzaley et al., 2008; Chadick et al., 2014). These findings would seem, at first blush, to contrast with the present results, in which older adults were generally successful in modulating encoding-related activity as a function of value. However, there are important differences between our paradigm and that used by Gazzaley et al. (2005). Most notably, participants in our task must generate retrieval cues for the to-be-remembered items in order to increase the probability that they will later be able to successfully free recall these items. It seems likely that this involves some sort of active process, such as linking semantic features of a given word with other to-be-remembered words. Participants can thus avoid being distracted by less relevant stimuli in a passive manner, by refraining from engaging semantic processing for a given word. This mechanism would seem to be very different from that evoked by the Gazzaley et al. studies, in which participants had to engage in active, top-down suppression of high-level visual processing for face or scene stimuli to avoid being distracted by attention-grabbing irrelevant stimuli (for a review of studies examining top-down suppression, see Zanto and Rissman, 2015).

\section{Age-related shifts in word-encoding period activity}

Despite general similarities in the spatial localization of behavioral and neuroimaging effects shown by older and younger adults in our data, we do find some clear differences. One reliable age difference is that, when examining areas in which value effects correlate with selectivity index, this association is reliably weaker in older adults in the most anterior portion of the pre-SMA cluster and the most ventral portion of the lateral PFC cluster. Instead, brain-behavior correlation effects seem to emerge more posteriorly in both of these areas in older adults (Fig. 3B). Notably, prior studies have found strong white matter connectivity between pre-SMA and lateral inferior PFC, particularly in the left hemisphere (e.g., Ford et al., 2010). This connection was recently 
defined as a distinct tract, called the frontal aslant tract (Catani et al., 2012). There is evidence that this tract is important in language production, as damage to it has been correlated with reductions in verbal fluency in aphasia patients (Catani et al., 2013). Other work has shown a rostrocaudal gradient of resting-state functional connectivity between pre-SMA and lateral PFC, such that posterior portions of pre-SMA are more strongly connected with posterior portions of lateral PFC, while anterior pre-SMA is connected with anterior portions of lateral PFC (Taren et al., 2011). There is also reason to believe that more posterior connections between these regions are more preserved with aging, as Ford et al. reported that anatomical connections measured by diffusion tractography between left posterior IFG and posterior medial PFC were stronger, relative to more anterior connections between these regions, in older adults than in young adults. Thus, the apparent anterior to posterior shift that we observe in pre-SMA may be part of an overall posterior shift in prefrontal activity in older adults.

The shifts in relevant prefrontal activity seem largely consistent with Rajah and D'Esposito (2005), at least in the general sense that the specific brain regions that are most effective for performing a task may be shifted in older adults due to the uneven rate at which agerelated degradation occurs in different prefrontal regions. Another potential aging-related shift in activity that has been described in previous studies is a reduction in hemispheric asymmetry in older adults (e.g., Cabeza, 2002). That pattern would suggest that while valuerelated differences in encoding-related activity in left hemisphere regions contribute to successful encoding in young adults, older adults might engage right hemisphere regions as well. We failed to find any evidence for such a reduction in laterality. In fact, young adults show a reliable tendency towards increased engagement of right prefrontal regions during encoding of high-value items, while older adults do not (Fig. 2B). Thus, our data suggest that older adults are showing, if anything, even stronger laterality than young adults in terms of how value affects encoding-related activity.

\section{Pre-stimulus (cue period) effects}

A striking difference between younger and older adults emerged during the value cue period, before the to-be-remembered word was presented. This difference is partially consistent with both the expectation deficit hypothesis proposed by Bollinger et al. (2011), and with the Dual Modes of Control framework (Braver et al., 2007; Braver, 2012). Bollinger et al. showed that older adults fail to show both the neural response and the behavioral benefit shown by young adults in response to a pre-stimulus cue that can help direct encoding. Braver et al. (2009; see also Paxton et al., 2008) showed that older adults tend to respond more reactively, while young adults respond more proactively, in a cognitive control task. Dew et al. (2012) subsequently showed that in a memory retrieval task, older adults tend to be less responsive than young adults to a cue that might lead to proactive retrieval-related processing, and instead show stronger, reactive activity in response to the stimulus itself, consistent with the Dual Modes of Control theory. The degree to which aging affects the timing of neural responses within a trial-during a pre-stimulus cue vs. during the stimulus-has not previously been examined in the context of memory encoding, however.

We find that both during the time period when the value cue is onscreen, and during the immediately following period when the word is on-screen, young adults show stronger engagement in regions related to semantic processing when the cue indicates a high point value. Older adults do not show these effects during the cue period, but they do show greater engagement of these critical semantic processing regions during the word-encoding period for high-value items. Thus, young adults are engaging PFC-mediated control mechanisms on high-value items both proactively and reactively. Older adults, on the other hand, seem to only respond reactively, waiting until they see the word that they need to encode before engaging in differential processing for high-value items.
At the same time, it is important to remember that the degree to which young adults selectively engage left prefrontal regions on highvalue items during the cue period does not seem to be associated with selectivity at recall. Thus, in this case, proactive encoding processes do not seem to be beneficial for young adults. This finding is in contrast to prior studies (Otten et al., 2006; Park and Rugg, 2010; Bollinger et al., 2010; Gruber and Otten, 2010; Addante et al., 2015) that have shown that items in which encoding-related processes are engaged proactively are more likely to be remembered on a later test. This could be due in part to the difference in our core analysis approach (i.e., acrosssubjects correlations between encoding activity and memory recall outcomes) versus that used in prior studies (i.e., within-subjects subsequent memory analysis). However, given that our analysis did reveal robust brain-behavior correlations during the word-encoding period, we believe it is more likely that the difference is attributable to the unique features of our task paradigm, which used multiple, relatively short lists and recall tests. We might predict that in other memory paradigms, age differences in the tendency to proactively engage encodingrelated activity in response to a motivationally-salient pre-stimulus cue could contribute to aging-related differences in memory, in line with the findings of Bollinger et al. (2011). The fact that there were still such aging-related differences in brain activity in the present context also suggests that increased proactive control in younger adults does not simply arise in situations where it can effectively enhance performance, but is also present even when it is seemingly inconsequential to task performance outcomes.

Another notable age difference between young and older adults is in how reward-related regions are activated during encoding. Young adults show strong differences in activity as a function of value both prior to and during encoding (cue period and word period), which are apparent in both the whole-brain analysis and when examining the network of reward-related regions defined as an ROI using the Neurosynth database. In contrast, for older adults, activity within these regions was not modulated by item value during either the cue or the word period. Interestingly, although the magnitude of value-related activity in the reward network ROI during the word period correlated with memory selectivity in young adults, differences in value-related modulation of activity in these same regions during the cue period were not at all associated with the degree to which value affected memory selectivity on the recall test in either age group.

This finding is in contrast to prior neuroimaging studies (e.g., Adcock et al., 2006; Gruber et al., 2014), in which successful memory of highvalue items was associated with greater activity in reward-sensitive regions prior to the appearance of the to-be-remembered item. As we note above in the context of pre-stimulus encoding effects, we cannot fully rule out the possibility that our use of a between-subjects assessment of subsequent memory, rather than a between-items assessment, accounts for differences between our study and prior work examining how value-related differences in brain activity in dopamine producing regions affects subsequent memory. However, we find two other major features of our study design to be more likely explanations. One is that our primary dependent measure was the degree to which people selectively recall high-value items on a free recall test, while prior studies have measured effects of value on recognition memory. In the procedure used here, subjects were required to recall items from successive lists, and became aware of the limited capacity of their recall ability and the need to be selective in order to maximize recall of valuable words. In this context, strategic efforts to modulate the degree to which semantic processing is engaged on high-value vs. low-value items may be a more effective way to enhance the relative memorability of high-value items than is anticipatory activation of a dopaminehippocampal circuit.

A second difference between our study and other studies of value effects on memory is that we used a very short delay between study and test, with the recall test typically beginning less than 1 min after encoding was completed for a given list. Adcock et al. (2006) tested 
memory after 24 h, and behavioral studies (e.g., Murayama and Kuhbandner, 2011; Spaniol et al., 2014) have demonstrated putatively dopamine-driven effects of value on memory performance that are present after a long delay of at least $24 \mathrm{~h}$, but are not reliably present on a test given at a short delay, up to 10 min after study. Other neuroimaging studies (Wolosin et al., 2012; Gruber et al., 2014) have found dopamine-driven effects of value on memory after a shorter delay, within a single experimental session. Still, even in those experiments, the average delay between study and test was longer than in our paradigm, with an average delay on the order of $20 \mathrm{~min}$ in both studies. Thus, it is possible that a longer delay would strengthen the degree to which effects of value on anticipatory activity in the dopamine system would relate to how strongly value affects subsequent memory. Additionally, given older adults' general insensitivity to value in dopaminergic brain regions in this study, it is possible that young adults would show a greater selectivity advantage over older adults with a long delay, compared to what we observe here.

Another notable difference between our study and prior work examining how reward affects memory is that our study uses points instead of monetary rewards to motivate encoding of high-value items. However, we did find that point value cues influenced activity in rewardsensitive brain regions in our young adult participants, suggesting that they are anticipating the possibility of future reward on high-value trials. We cannot rule out the possibility that older adults are treating point-based rewards differently than they would treat monetary rewards, but given that they do still recall far more high-value items than low-value items, there is clearly a high degree of motivational salience to the point values that we provide.

It is not clear whether the lack of value-related differences in the proactive engagement of brain regions related to semantic control and reward processing is due to a strategic choice by older adults, which would suggest greater efficiency in older adults than in young adults, or whether it is due to older adults having a reduced ability to spontaneously engage encoding processes proactively. Either way, it seems that young adults may not be proactively recruiting encoding processes in a controlled way to enhance performance, but instead engage in such processes whether or not they are effective. It may be that in young adults, the engagement of proactive control processes is automatic, and not in response to task demands.

However, prior work (e.g., Braver et al., 2009; Bollinger et al., 2011; Dew et al., 2012) has also shown that older adults tend to not respond proactively to stimuli even when such responding is helpful. Thus, it seems likely that older adults' relatively preserved ability to control memory encoding in the value-directed remembering paradigm is related to the ineffectiveness of proactive modulation of encodingrelated mechanisms in this paradigm. We can then assume that older adults would likely show a greater decrement in performance than what we observe here if proactive responding to the coming stimulus were effective in achieving the task goals.

Relatedly, it is notable that the primary effective mechanism in achieving memory selectivity, value-modulated engagement of a left-lateralized fronto-temporal network associated with semantic encoding, is essentially preserved across age groups. This preservation across age groups of the most effective mechanism for controlling encoding as a function of value is likely another key piece of the puzzle as to why older adults are able to achieve largely comparable levels of memory selectivity to young adults. That is, in addition to not employing the mechanisms that appear to be ineffective in young adults, namely, proactive engagement of semantic and reward processing regions, older adults are able to successfully implement the strategy that is effective across both age groups, reactive engagement of semantic processing. Thus, it may be that to optimize the efficiency of memory performance in older adults, they should be given the opportunity to selectively and reactively engage semantic encoding processes for important information.

\section{Acknowledgments}

This work was supported by the Scientific Research Network for Decision Neuroscience and Aging (SRNDNA), as a subaward under National Institutes of Health grant AG039350 (to B.J.K., M.S.C., J.R., and A.D.C.); the National Institute on Aging at the National Institutes of Health (grant F31 AG047048 to M.S.C., grant R01 AG044035 to A.D.C.); the National Science Foundation (grant BCS-0848246 to B.J.K.); the UCLA Staglin Center for Cognitive Neuroscience (pilot funds awarded to B.J.K.); and a UCLA Academic Senate Faculty Research Grant to B.J.K. We thank Saskia Giebl for assistance with recruiting and running participants, Garth Carlson for publicizing our recruitment materials, as well as Aimee Drolet Rossi and Gregory Samanez-Larkin for suggestions related to task design.

\section{Appendix A. Supplementary data}

Supplementary data to this article can be found online at http://dx. doi.org/10.1016/j.neuroimage.2015.07.079.

\section{References}

Adcock, R.A., Thangavel, A., Whitfield-Gabrieli, S., Knutson, B., Gabrieli, J.D.E., 2006. Reward-motivated learning: mesolimbic activation precedes memory formation. Neuron 50, 507-517.

Addante, R.J., de Chastelaine, M., Rugg, M.D., 2015. Pre-stimulus neural activity predicts successful encoding of inter-item associations. Neuroimage 105, 21-31.

Addis, D.R., McAndrews, M.P., 2006. Prefrontal and hippocampal contributions to the generation and binding of semantic associations during successful encoding. Neuroimage 33, 1194-1206.

Ariel, R., Castel, A.D., 2014. Eyes wide open: enhanced pupil dilation when selectivity studying important information. Exp. Brain Res. 232, 337-344.

Binder, J.R., Desai, R.H., 2011. The neurobiology of semantic memory. Trends Cogn. Sci. 15, 527-536.

Binder, J.R., Desai, R.H., Graves, W.W., Conant, L.L., 2009. Where is the semantic system? A critical review and meta-analysis of 120 functional neuroimaging studies. Cereb. Cortex 19, 2767-2796.

Bollinger, J., Rubens, M.T., Zanto, T.P., Gazzaley, A., 2010. Expectation-driven changes in cortical functional connectivity influence working memory and long-term memory performance. J. Neurosci. 30, 14399-14410.

Bollinger, J., Rubens, M.T., Masangkay, E., Kalkstein, J., Gazzaley, A., 2011. An expectationbased memory deficit in aging. Neuropsychologia 49, 1466-1475.

Braver, T.S., 2012. The variable nature of cognitive control: a dual mechanisms framework. Trends Cogn. Sci. 16, 106-113.

Braver, T.S., Gray, J.R., Burgess, G.C., 2007. Explaining the many varieties of working memory variation: dual mechanisms of cognitive control. In: Conway, A.R.A., Jarrold, C., Kane, M.J., Miyake, A., Towse, J.N. (Eds.), Variation in Working Memory. Oxford University Press, New York, pp. 76-106.

Braver, T.S., Paxton, J.L., Locke, H.S., Barch, D.M., 2009. Flexible neural mechanisms of cognitive control within human prefrontal cortex. Proc. Natl. Acad. Sci. 106, 7351-7356.

Brodmann, K., 1909. Vegleichende Lokalisationslehre der Grosshirnde. Barth, Leipzig (Germany).

Bunzeck, N., Schutze, H., Stallforth, S., Kaufmann, J., Düzel, S., Heinze, H.J., Düzel, E., 2007. Mesolimbic novelty processing in older adults. Cereb. Cortex 17, 2940-2948.

Bunzeck, N., Doeller, C.F., Dolan, R.J., Düzel, E., 2012. Contextual interaction between novelty and reward processing within the mesolimbic system. Hum. Brain Mapp. 33, 1309-1324.

Cabeza, R., 2002. Hemispheric asymmetry reduction in older adults: the HAROLD Model. Psychol. Aging 17, 85-100.

Cabeza, R., Anderson, N.D., Locantore, J.K., McIntosh, A.R., 2002. Aging gracefully: compensatory brain activity in high-performing older adults. Neuroimage 17, 1394-1402.

Castel, A.D., 2008. The adaptive and strategic use of memory by older adults: evaluative processing and value-directed remembering. In: Benjamin, A.S., Ross, B.H. (Eds.), The Psychology of Learning and Motivation Vol. 48. Academic Press, San Diego, pp. 225-270.

Castel, A.D., Benjamin, A.S., Craik, F.I.M., Watkins, M.J., 2002. The effects of aging on selectivity and control in short-term recall. Mem. Cogn. 30, 1078-1085.

Castel, A.D., Farb, N.A.S., Craik, F.I.M., 2007. Memory for general and specific value information in younger and older adults: Measuring the limits of strategic control. Mem. Cogn. 35, 685-700.

Castel, A.D., Balota, D.A., McCabe, D.P., 2009. Memory efficiency and the strategic control of attention at encoding: impairments of value-directed remembering in Alzheimer's disease. Neuropsychology 23, 297-306.

Catani, M., Dell'acqua, F., Vergani, F., Malik, F., Hodge, H., Roy, P., Valabregue, R., de Schotten, M.T., 2012. Short frontal lobe connections of the human brain. Cortex 48, 273-291.

Catani, M., Mesulam, M.M., Jakobsen, E., Malik, F., Martersteck, A., Wieneke, C., Thompson, C.K., de Schotten, M.T., Dell'Acqua, F., Weintraub, S., Rogalski, E., 2013. A novel frontal 
pathway underlies verbal fluency in primary progressive aphasia. Brain 136, 2619-2628.

Chadick, J.Z., Zanto, T.P., Gazzaley, A., 2014. Structural and functional differences in prefrontal cortex underlie distractibility and suppression deficits in ageing. Nat. Commun. 5, 1-12.

Cohen, M.S., Rissman, J., Suthana, N.A., Castel, A.D., Knowlton, B.J., 2014. Value-based modulation of memory encoding involves strategic engagement of fronto-temporal semantic processing regions. Cogn. Affect. Behav. Neurosci. 14, 578-592.

Crosson, B., Sadek, J.R., Maron, L., Gökçay, D., Mohr, C.M., Auerbach, E.J., Freeman, A.J., Leonard, C.M., Briggs, R.W., 2001. Relative shift in activity from medial to lateral frontal cortex during internally versus externally guided word generation. J. Cogn. Neurosci. 13, 272-283.

Daselaar, S.M., Prince, S.E., Cabeza, R., 2004. When less means more: deactivations during encoding that predict subsequent memory. Neuroimage 23, 921-927.

Dew, I.T.Z., Buchler, N., Dobbins, I.G., Cabeza, R., 2012. Where is ELSA? The early to late shift in aging. Cereb. Cortex 22, 2542-2553.

Duverne, S., Motamedinia, S., Rugg, M.D., 2009. The relationship between aging, performance, and the neural correlates of successful memory encoding. Cereb. Cortex 19, 733-744.

Düzel, E., Bunzeck, N., Guitart-Masip, M., Düzel, S., 2010. NOvelty-related motivation of anticipation and exploration by dopamine (NOMAD): implications for healthy aging. Neurosci. Biobehav. Rev. 34, 660-669.

Folstein, M.F., Folstein, S.E., McHugh, P.R., 1975. Mini-mental state: a practical method for grading the cognitive state of patients for the clinician. J. Psychiatr. Res. 12, 189-198.

Ford, A., McGregor, K.M., Case, K., Crosson, B., White, K.D., 2010. Structural connectivity of Broca's area and medial frontal cortex. Neuroimage 52, 1230-1237.

Galli, G., 2014. What makes deeply encoded items memorable? Insights into the levels of processing framework from neuroimaging and neuromodulation. Front. Psychol. 5, 61.

Gazzaley, A., Cooney, J.W., Rissman, J., D'Esposito, M., 2005. Top-down suppression deficit underlies working memory impairment in normal aging. Nat. Neurosci. $8,1298-1300$.

Gazzaley, A., Clapp, W., McEvoy, K., Knight, R., D'Esposito, M., 2008. Age-related top-down suppression deficit in the early stages of cortical visual memory processing. Proc. Natl. Acad. Sci. 105, 13122-13126.

Gruber, M.J., Otten, L.J., 2010. Voluntary control over prestimulus activity related to encoding. J. Neurosci. 30, 9793-9800.

Gruber, M.J., Gelman, B.D., Ranganath, C., 2014. States of curiosity modulate hippocampus-dependent learning via the dopaminergic circuit. Neuron 84, 486-496.

Hayes, M.G., Kelly, A.J., Smith, A.D., 2013. Working memory and the strategic control of attention in older and younger adults. J. Gerontol. B Psychol. Sci. Soc. Sci. 68, 176-183.

Holm, S., 1979. A simple sequentially rejective multiple test procedure. Scand. J. Stat. 6, 65-70.

Jenkinson, M., Bannister, P., Brady, M., Smith, S., 2002. Improved optimization for the robust and accurate linear registration and motion correction of brain images. Neuroimage 17, 825-841.

Kirchhoff, B.A., Buckner, R.L., 2006. Functional-anatomic correlates of individual differences in memory. Neuron 51, 263-274.

Kirchhoff, B.A., Anderson, B.A., Barch, D.M., Jacoby, L.L., 2012. Cognitive and neural effects of semantic encoding strategy training in older adults. Cereb. Cortex 22, 788-799.

Lancaster, J.L., Tordesillas-Gutiérrez, D., Martinez, M., Salinas, F., Evans, A., Zilles, K. Mazziotta, J.C., Fox, P.T., 2007. Bias between MNI and Talairach coordinates analyzed using the ICBM-152 brain template. Hum. Brain Mapp. 28, 1194-1205.

Li, S.-C., Lindenberger, U., Sikström, S., 2001. Aging cognition: from neuromodulation to representation. Trends Cogn. Sci. 5, 479-486.

Li, S., Cullen, W.K., Anwyl, R., Rowan, M.J., 2003. Dopamine-dependent facilitation of LTP induction in hippocampal CA1 by exposure to spatial novelty. Nat. Neurosci. 6, $526-531$.

Logan, J.M., Sanders, A.L., Snyder, A.Z., Morris, J.C., Buckner, R.L., 2002. Under-recruitment and nonselective recruitment: dissociable neural mechanisms associated with aging. Neuron 33, 827-840.

Mattson, J.T., Wang, T.H., de Chastelaine, M., Rugg, M.D., 2015. Effects of age on negative subsequent memory effects associated with the encoding of item and item-context information. Cereb. Cortex 24, 3322-3333.

McGillivray, S., Castel, A.D., 2011. Betting on memory leads to metacognitive improvement in younger and older adults. Psychol. Aging 26, 137-142.

Miotto, E.C., Savage, C.R., Evans, J.J., Wilson, B.A., Martins, M.G., Iaki, S., Amaro Jr., E., 2006. Bilateral activation of the prefrontal cortex after strategic semantic cognitive training. Hum. Brain Mapp. 27, 288-295.

Miotto, E.C., Balardin, J.B., Savage, C.R., Martin, M., Batistuzzo, M.C., Amaro Jr., E., Nitrini, R., 2014. Brain regions supporting verbal memory improvement in healthy older subjects. Arq. Neuropsiquiatr. 72, 663-670.

Murayama, K., Kitagami, S., 2014. Consolidation power of extrinsic rewards: reward cues enhance long-term memory for irrelevant past events. J. Exp. Psychol. Gen. 143, $15-20$.

Murayama, K., Kuhbandner, C., 2011. Money enhances memory consolidation-but only for boring material. Cognition 119, 120-124.
Otten, L.J., Rugg, M.D., 2001. When more means less: neural activity related to unsuccessful memory encoding. Curr. Biol. 11, 1528-1530.

Otten, L.J., Quayle, A.H., Akram, S., Ditewig, T.A., Rugg, M.D., 2006. Brain activity before an event predicts later recollection. Nat. Neurosci. 9, 489-491.

Paller, K.A., Wagner, A.D., 2002. Observing the transformation of experience into memory. Trends Cogn. Sci. 6, 93-102.

Park, H., Rugg, M.D., 2010. Prestimulus hippocampal activity predicts later recollection. Hippocampus 20, 24-28.

Paxton, J.L., Barch, D.M., Racine, C.A., Braver, T.S., 2008. Cognitive control goal maintenance and prefrontal function in healthy aging. Cereb. Cortex 18, 1010-1028.

Persson, J., Lustig, C., Nelson, J.K., Reuter-Lorenz, P.A., 2007. Age differences in deactivation: a link to cognitive control. J. Cogn. Neurosci. 19, 1021-1032.

Raichle, M.E., MacLeod, A.M., Snyder, A.Z., Powers, W.J., Gusnard, D.A., Shulman, G.L., 2001. A default mode of brain function. Proc. Natl. Acad. Sci. 98, 676-682.

Rajah, M.N., D'Esposito, M., 2005. Region-specific changes in prefrontal function with age: a review of PET and fMRI studies on working and episodic memory. Brain 128 , 1964-1983.

Revelle, W., 2015. Psych: procedures for personality and psychological research. http:// CRAN.R-project.org $/$ package $=$ psych

Rosen, A.C., Prull, M.W., O'Hara, R., Race, E.A., Desmond, J.E., Glover, G.H., Yesavage, J.A. Gabrieli, J.D.E., 2002. Variable effects of aging on frontal lobe contributions to memory. Neuroreport 13, 2425-2428.

Rossi, S., Miniussi, C., Pasqualetti, P., Babiloni, C., Rossini, P.M., Cappa, S.F., 2004. Agerelated functional changes of prefrontal cortex in long-term memory: a repetitive transcranial magnetic stimulation study. J. Neurosci. 24, 7939-7944.

Samanez-Larkin, G.R., D'Esposito, M., 2008. Group comparisons: imaging the aging brain Soc. Cogn. Affect. Neurosci. 3, 290-297.

Savage, C.R., Deckersbach, T., Heckers, S., Wagner, A.D., Schacter, D.L., Alpert, N.M., Rauch, S.L., 2001. Prefrontal regions supporting spontaneous and directed application of verbal learning strategies: evidence from PET. Brain 124, 219-231.

Shohamy, D., Adcock, R.A., 2010. Dopamine and adaptive memory. Trends Cogn. Sci. 14 464-472.

Smith, S.M., 2002. Fast robust automated brain extraction. Hum. Brain Mapp. 17, $143-155$.

Spaniol, J., Schain, C., Bowen, H.J., 2014. Reward-enhanced memory in younger and older adults. J. Gerontol. B Psychol. Sci. Soc. Sci. 69, 730-740.

Steiger, J.H., 1980. Tests for comparing elements of a correlation matrix. Psychol. Bull. 87 245-251.

Talairach, J., Tournoux, P., 1988. Co-planar stereotaxic atlas of the human brain. Thieme, New York.

Taren, A.A., Venkatraman, V., Huettel, S.A., 2011. A parallel functional topography between medial and lateral prefrontal cortex: Evidence and implications for cognitive control. J. Neurosci. 31, 5026-5031.

Toglia, M.P., Battig, W.F., 1978. Handbook of Semantic Word Norms. Erlbaum, Hillsdale N (Republished online as a supplement to Toglia M. P. 2009. Withstanding the test of time: The 1978 semantic word norms. Behav Res Methods. 41:531-533).

Tremblay, P., Gracco, V.L., 2006. Contribution of the frontal lobe to externally and internally specified verbal responses: fMRI evidence. Neuroimage 33, 947-957.

Van Essen, D.C., Drury, H.A., Dickson, J., Harwell, J., Hanlon, D., Anderson, C.H., 2001. An integrated software suite for surface-based analyses of cerebral cortex. J. Am. Med. Inform. Assoc. 8, 443-459.

Van Essen, D.C., Glasser, M.F., Dierker, D.L., Harwell, J., Coalson, T., 2012. Parcellations and hemispheric asymmetries of human cerebral cortex analyzed on surface-based atlases. Cereb. Cortex 22, 2241-2262.

Verhaeghen, P., Cerella, J., 2002. Aging executive control and attention: a review of metaanalyses. Neurosci. Biobehav. Rev. 26, 849-857.

Wagner A.D., Schacter, D.L, Rotte, M., Koutstaal, W., Maril, A, Dale, A.M., Rosen, B.R Buckner, R.L., 1998. Building memories: remembering and forgetting of verbal experiences as predicted by brain activity. Science 288, 1188-1191.

Wagner, A.D., Paré-Blagoev, E.J., Clark, J., Poldrack, R.A., 2001. Recovering meaning: left prefrontal cortex guides controlled semantic retrieval. Neuron 31, 329-338.

Watkins, M.J., Bloom, L.C., 1999. Selectivity in memory: An exploration of willful contro over the remembering process. Unpublished manuscript.

Whitney, C., Kirk, K., O'Sullivan, J., Lambon Ralph, M.A., Jefferies, E., 2011. The neural organization of semantic control: TMS evidence for a distributed network in left inferior frontal and posterior middle temporal gyrus. Cereb. Cortex 21, 1066-1075.

Wolosin, S.M., Zeithamova, D., Preston, A.R., 2012. Reward modulation of hippocampa subfield activation during successful associative encoding and retrieval. J. Cogn. Neurosci. 24, 1532-1547.

Woolrich, M.W., Ripley, B.D., Brady, J.M., Smith, S.M., 2001. Temporal autocorrelation in univariate linear modelling of fMRI data. Neuroimage 14, 1370-1386.

Yarkoni, T., Poldrack, R.A., Nichols, T.E., Van Essen, D.C., Wager, T.D., 2011. Large-scale automated synthesis of human functional neuroimaging data. Nat. Methods 8 , 665-670.

Zanto, T., Rissman, J., 2015. Top-down suppression. In: Toga, A.W. (Ed.)Brain Mapping: An Encyclopedic Reference vol. 3. Academic Press: Elsevier, pp. 261-267. 